\title{
Galaxy cluster searches based on photometric redshifts in the four CFHTLS Wide fields ${ }^{\star}, \star \star$
}

\author{
F. Durret ${ }^{1,2}$, C. Adami $^{3}$, A. Cappi ${ }^{4,5}$, S. Maurogordato ${ }^{5}$, I. Márquez ${ }^{6}$, O. Ilbert $^{3}$, J. Coupon ${ }^{1,2,7}$, S. Arnouts ${ }^{3,8}$, \\ C. Benoist ${ }^{5}$, J. Blaizot ${ }^{9}$, T. M. Edorh ${ }^{10}$, B. Garilli ${ }^{3,11}$, L. Guennou ${ }^{3}$, V. Le Brun ${ }^{3}$, O. Le Fèvre ${ }^{3}$, A. Mazure ${ }^{3}$, \\ H. J. McCracken ${ }^{1,2}$, Y. Mellier ${ }^{1,2}$, C. Mezrag ${ }^{12}$, E. Slezak ${ }^{5}$, L. Tresse ${ }^{3}$, and M. P. Ulmer ${ }^{13}$ \\ ${ }^{1}$ UPMC Université Paris 06, UMR 7095, Institut d'Astrophysique de Paris, 98bis Bd Arago, 75014 Paris, France \\ e-mail: durret@iap.fr \\ 2 CNRS, UMR 7095, Institut d'Astrophysique de Paris, 75014 Paris, France \\ ${ }^{3}$ LAM, OAMP, Pôle de l'Étoile Site Château-Gombert, 38 rue Frédéric Juliot-Curie, 13388 Marseille Cedex 13, France \\ 4 INAF - Osservatorio Astronomico di Bologna, via Ranzani 1, 40127 Bologna, Italy \\ 5 OCA, Cassiopée, Boulevard de l'Observatoire, BP 4229, 06304 Nice Cedex 4, France \\ ${ }^{6}$ Instituto de Astrofísica de Andalucía (CSIC), Apdo. 3004, 18080 Granada, Spain \\ 7 Astronomical Institute, Graduate School of Science, Tohoku University Sendai 980-8578, Japan \\ ${ }^{8}$ Canada-France-Hawaii Telescope Corporation, Kamuela, HI 96743, USA \\ 9 CRAL (UMR 5574), Université Claude Bernard Lyon 1 (UCBL), École Normale Supérieure de Lyon (ENS-L), and Centre National \\ de la Recherche Scientifique (CNRS), 69230 Saint-Genis Laval, France \\ ${ }^{10}$ Université Paris 07 Denis Diderot, 75205 Paris Cedex, France \\ 11 INAF IASF - Milano, via Bassini 15, 20133 Milano, Italy \\ 12 ENS-Cachan, 61 avenue du président Wilson, 94235 Cachan Cedex, France \\ 13 Department of Physics \& Astronomy, Northwestern University, Evanston, IL 60208-2900, USA
}

Received 30 March 2011 / Accepted 1 September 2011

\begin{abstract}
Context. Cosmological parameters can be constrained by counting clusters of galaxies as a function of mass and redshift and by considering regions of the sky sampled as deeply and as homogeneously as possible.

Aims. Several methods for detecting clusters in large imaging surveys have been developed, among which the one used here, which is based on detecting structures. This method was first applied to the Canada France Hawaii Telescope Legacy Survey (CFHTLS) Deep 1 field by Mazure et al. (2007, A\&A, 467, 49), then to all the Deep and Wide CFHTLS fields available in the T0004 data release by Adami et al. (2010, A\&A, 509, A81). The validity of the cluster detection rate was estimated by applying the same procedure to galaxies from the Millennium simulation. Here we use the same method to analyse the full CFHTLS Wide survey, based on the T0006 data release.

Methods. Our method is based on the photometric redshifts computed with Le Phare for all the galaxies detected in the Wide fields, limited to magnitudes $z^{\prime} \leq 22.5$. We constructed galaxy density maps in photometric redshift bins of 0.1 based on an adaptive kernel technique, detected structures with SExtractor at various detection levels, and built cluster catalogues by applying a minimal spanning tree algorithm.

Results. In a total area of $154 \mathrm{deg}^{2}$, we have detected 4061 candidate clusters at $3 \sigma$ or above (6802 at $2 \sigma$ and above), in the redshift range $0.1 \leq z \leq 1.15$, with estimated mean masses between $1.3 \times 10^{14}$ and $12.6 \times 10^{14} M_{\odot}$. This catalogue of candidate clusters will be available at the CDS. We compare our detections with those made in various CFHTLS analyses with other methods. By stacking a subsample of clusters, we show that this subsample has typical cluster characteristics (colour-magnitude relation, galaxy luminosity function). We also confirm that the cluster-cluster correlation function is comparable to the one obtained for other cluster surveys and analyse large-scale filamentary galaxy distributions.

Conclusions. We have increased the number of known optical high-redshift cluster candidates by a large factor, an important step towards obtaining reliable cluster counts to measure cosmological parameters. The clusters that we detect behave as expected if they are located at the intersection of filaments by which they are fed.
\end{abstract}

Key words. large-scale structure of Universe - galaxies: clusters: general - surveys

\footnotetext{
* Based on observations obtained with MegaPrime/MegaCam, a joint project of CFHT and CEA/DAPNIA, at the Canada-France-Hawaii Telescope (CFHT) which is operated by the National Research Council (NRC) of Canada, the Institut National des Sciences de l'Univers of the Centre National de la Recherche Scientifique (CNRS) of France, and the University of Hawaii. This work is based on data products produced at TERAPIX and the Canadian Astronomy Data Centre as part of the CFHT Legacy Survey, a collaborative project of the NRC and CNRS.

$\star \star$ The catalog of candidate clusters is available at the CDS via anonymous ftp to cdsarc.u-strasbg.fr $(130.79 .128 .5)$ or via http://cdsarc.u-strasbg.fr/viz-bin/qcat?J/A+A/535/A65
}

\section{Introduction}

The cluster count technique (e.g., Gioia et al. 1990) allows putting strong constraints on cosmological parameters, but requires catalogues with large numbers of clusters at high redshift $(z \geq 1)$ and in extended fields of view (several tens of square degrees). Many large surveys have been done in the past ten years. One of their aims was to obtain large catalogues of galaxy clusters, among these the Canada France Hawaii Telescope Legacy 
Survey (CFHTLS) ${ }^{1}$. The CFHTLS observations are carried out in five filters $\left(u^{*}, g^{\prime}, r^{\prime}, i^{\prime}\right.$ or $y$, and $\left.z^{\prime}\right)$ providing catalogues of sources that are $80 \%$ complete up to $i_{\mathrm{AB}}=24.0$ for the CFHTLS Wide (see Goranova et al. 2009). The CFHTLS survey encloses a sample of about $20 \times 10^{6}$ galaxies in a volume of about $1 \mathrm{Gpc}^{3}$, with a median redshift of $z \sim 0.60$ within a limiting magnitude $i^{\prime} \leq 23$ for the Wide survey (see Table 6 in Coupon et al. 2009). Romer et al. (2001) estimate that about 20000 clusters with temperatures $k T>2 \mathrm{keV}$ and redshifts $z \leq 1.5$ were expected in $800 \mathrm{deg}^{2}$ assuming a standard cosmological model. The corresponding number of clusters expected in the $154 \mathrm{deg}^{2}$ covered by the CFHTLS (not including the Deep survey) would then be 3850 , consistent with the number of cluster candidates we found. We are indeed considering comparable mass ranges such as Romer et al. (2001): our $3 \sigma$ detections correspond to a minimal mass of $1.3 \times 10^{13} M_{\odot}$ and to a mean mass of $1.8 \times 10^{14} M_{\odot}$ (see Table 2), while the scaling relation shown by Juett et al. (2010) indicates that clusters with $k T>2 \mathrm{keV}$ have masses of $M_{500} E(z)>10^{14} M_{\odot}$.

Early searches for clusters of galaxies in the CFHTLS were performed by Olsen et al. (2007, and 2008) and Grove et al. (2009), based on a matched filter detection algorithm applied to the Deep fields. An improvement of this technique has recently been developed by Milkeraitis et al. (2010) and also applied to the CFHTLS Deep fields. Lensing techniques were employed to detect massive structures (i.e. with masses over $10^{13} M_{\odot}$ ) in the CFHTLS (e.g., Cabanac et al. 2007; Gavazzi \& Soucail 2007; Bergé et al. 2008). Other cluster studies based on the CFHTLS data (e.g., the CFHTLS-CARS survey: Erben et al. 2009; Hildebrandt et al. 2009) and based in part on the red sequence in the colour magnitude diagram have also been developed. Bielby et al. (2010) have recently identified high-redshift $(z>1.1)$ groups and clusters in the Deep 1 and Deep 4 fields, by combining CFHTLS optical data with the WIRcam Deep Survey (WIRDS) in the infrared and XMM-Newton data. Ascaso et al. (2010) applied a Bayesian cluster finder to detect galaxy clusters in several surveys including the CFHTLS, and have detected $90 \%$ of the clusters found by Olsen et al. (2007) and Adami et al. (2010, hereafter A10).

We have developed a new method to search for clusters in large multiband imaging surveys and applied it to the CFHTLS. The first results were presented by Mazure et al. (2007) for the CFHTLS Deep 1 field. More extensive results were later obtained by A10 for the CFHTLS Deep 2, Deep 3 and Deep 4 fields, as well as for the CFHTLS W1, W3, and W4, as available in 2008 (T0004 data release). A10 also applied the same method to the Millennium simulation, in order to assess the validity of the method and results. No other data have been obtained for the Deep fields since then, so we will not discuss these fields any further. On the other hand, the CFHTLS is now complete and the coverage of the Wide fields has become much broader in the T0006 data release, leading us to reanalyse all the Wide fields in a consistent way.

Thanjavur et al. (2009) developed another method of detecting galaxy clusters named $\mathrm{K} 2$, which they applied to the CFHTLS Wide fields (T0005 data release). This method is based on the red sequence, and it detects cluster enhancements in both colours and position. Since these authors have kindly made their catalogues available to us, we will be able to compare our list of cluster candidates directly with theirs (see Sect. 4).

Van Breukelen \& Clewley (2009) have developed yet another algorithm, named $2 \mathrm{TecX}$, to search for high-redshift clusters

\footnotetext{
${ }^{1}$ http://www.cfht.hawaii.edu/Science/CFHLS/
}

in optical/infrared imaging surveys. This method is based on photometric redshifts estimated from the full redshift probability function and on identifying cluster candidates through crosschecking two different selection techniques (adaptations of the Voronoi tesselations and of the friends-of-friends method). This method is not all that different from ours, and it would be very interesting to apply it to the CFHTLS Wide data, to compare the cluster candidates obtained by their method and by ours. A comparable method has been applied to the SDSS Stripe 82 by Geach et al. (2011) to search for clusters up to $z \sim 0.6$.

The paper is organized as follows. The method of searching for clusters is described in Sect. 2. Results for cluster candidates are described in Sect. 3: numbers, spatial distribution, redshift distribution. Full tables of our cluster detections are available electronically. In Sect. 4 we compare our cluster candidates to those found by other authors. The angular correlation function is discussed in Sect. 6. We discuss the cosmological implications of our work in Sect. 7 and conclude in Sect. 8.

In this paper we assume $H_{0}=70 \mathrm{~km} \mathrm{~s}^{-1} \mathrm{Mpc}^{-1}, \Omega_{\mathrm{m}}=0.3$, and $\Omega_{\Lambda}=0.7$. All coordinates are given at the J2000 equinox and magnitudes are in the $\mathrm{AB}$ system.

\section{The method to search for clusters in the CFHTLS}

We adopt here the same method as in M07 and A10, where a full description is given. As mentioned above, we have redone the analysis for the four full Wide fields, for which the data are now much more extensive and of better quality than those available in the T0004 data release on which the A10 paper was based: the total surface covered by the CFHTLS data is now about $154 \mathrm{deg}^{2}$, which is about 4.4 times more extended than the $35 \mathrm{deg}^{2}$ covered by the T0004 data release, the photometric zero points were homogenized throughout the entire Wide survey, and the spectroscopic sample was larger, thus allowing more accurate computations of the photometric redshifts.

Our approach is based on photometric redshifts computed for all the galaxies extracted in each field (Coupon et al. 2009), taking the full colour information into account. We divide the galaxy catalogues in slices of 0.1 in redshift, each slice overlapping the previous one by 0.05 , and build galaxy density maps for each redshift slice. Structures in these density maps are detected with the SExtractor software (Bertin \& Arnouts 1996) in the different redshift bins at various significance levels. We then analyse possible substructuring by applying a minimal spanning tree algorithm to our data. In order to assess the validity of this method, the same treatment was applied by A10 to the Millennium simulation, and we refer the reader to this paper.

\subsection{Photometric redshifts}

The Wide fields are mosaics of $1 \times 1 \mathrm{deg}^{2}$ Megacam fields observed in the $u^{*}, g^{\prime}, r^{\prime}, i^{\prime}$, and $z^{\prime}$ bands. Our approach is based on photometric redshifts, which can be estimated with good precision up to $z \sim 1.2$ (Mellier et al. 2008; Coupon et al. 2009). Photometric redshifts were computed for all the objects in the CFHTLS galaxy catalogues of the data release T0006 with the Le Phare software developed by S. Arnouts and O. Ilbert (Ilbert et al. 2006; also see http://www.ifa.hawaii.edu/ ilbert/these.pdf.gz, pp. 50 and 142). They were optimized with spectroscopic redshifts from the literature, including the VVDS (e.g., Le Fèvre et al. 2005). The resulting statistical errors on the photometric redshifts $\left(\sigma_{\Delta z /(1+z s)}\right)$ are given in Coupon et al. (2009). For example in the W1 field, they continuously increase (between $i^{\prime}=20.5$ and $i^{\prime}=24$ ) from 0.025 to 


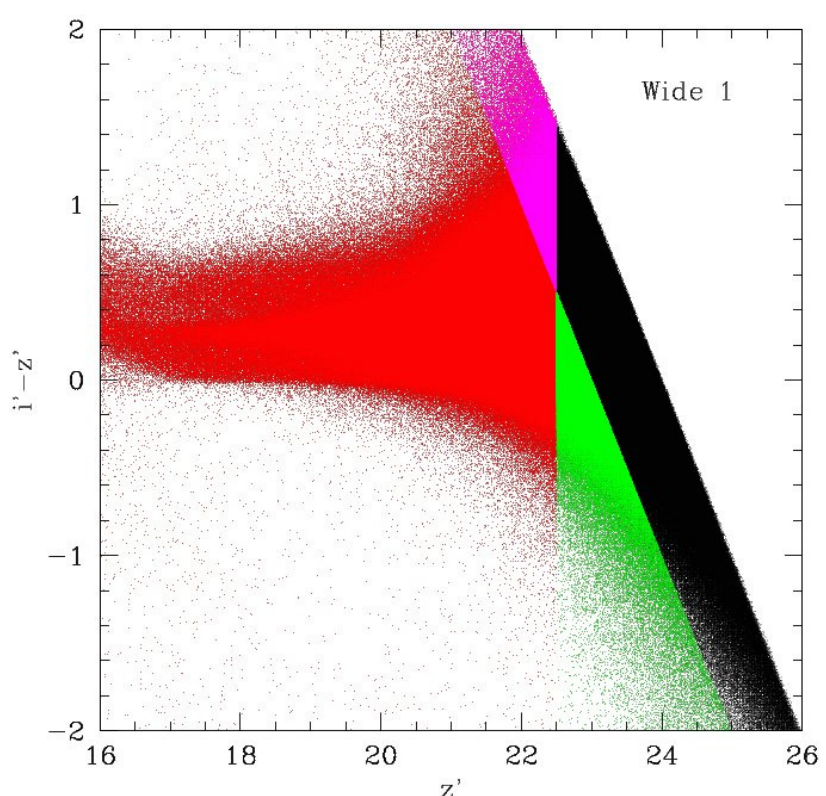

Fig. 1. $i^{\prime}-z^{\prime}$ versus $z^{\prime}$ colour magnitude diagram for galaxies in the Wide 1 field. Black points represent the entire galaxy sample. Red+magenta dots are the galaxies corresponding to our present selection $\left(z^{\prime} \leq 22.5\right.$ and $0.1 \leq z$ phot $\left.\leq 1.15\right)$. Magenta dots are objects included with our present selection that would not have been included with the $i^{\prime} \leq 23$ selection of A10. Green dots are objects included by the A10 selection but not by the present selection. The limit to the black points is due to a magnitude cut in the initial catalogue of galaxy photometric redshifts at $i^{\prime}<24$ or $y<24$ (depending on the $i$ filter available).

0.053. At our limiting magnitude of $z^{\prime}=22.5$ (roughly corresponding to $i^{\prime}=23$ ), the statistical error on the redshift is 0.043 . Extensive tests of photometric redshift software performed by Hildebrandt et al. (2010) have demonstrated the excellent results of the Le Phare technique.

To avoid incompleteness effects and strong systematic biases in photometric redshift computations, the galaxy catalogues were limited to $z^{\prime} \leq 22.5$. Galaxy magnitude histograms show that this limit is located $\sim 0.5 \mathrm{mag}$ below the value where incompleteness begins to show, therefore our analysis should not suffer from incompleteness effects. In our previous analysis of the Wide fields, we limited our catalogues to $i^{\prime} \leq 23$, but we could not apply this condition in the present work because the $i^{\prime}$ filter had to be replaced by a new similar (but not identical) filter $y$ between data releases T0004 and T0006, so a selection in the $i^{\prime}$ band would no longer be homogeneous. The T0004 data in the $i^{\prime}$ band have not been discarded but merged into the T0006 data.

To test the consistency of the present $z^{\prime} \leq 22.5$ selection with the previous $i^{\prime} \leq 23$ selection, we show in Fig. 1 the $i^{\prime}-z^{\prime}$ versus $z^{\prime}$ colour magnitude diagram for galaxies in the Wide 1 field. The plots for the three other Wide fields are similar. This plot illustrates that by considering a sample with $z^{\prime} \leq 22.5$ we are including the magenta points (which would not have included by the $y \leq 23$ condition) and losing the green points (those with $y \leq 23$ but $z^{\prime}>22.5$ ). The numbers of points corresponding to each criterium are given in Table 1 for the four Wide fields. By applying the $z^{\prime} \leq 22.5$ condition, we therefore increase the number of galaxies by $4 \%$ to $5 \%$ and decrease it by $14 \%$ to $19 \%$ relative to the $y \leq 23$ condition.

We selected galaxies with photometric redshifts included in the range $0.1 \leq z \leq 1.15$. For each CFHTLS Wide field, we give in Table 1 the numbers of galaxies considered (i.e. with
Table 1. Numbers of points corresponding to the three different selections illustrated in Fig. 1 for the four Wide fields, and fractions of "gained" and "lost" points due to the change in selection criterium.

\begin{tabular}{lcccc}
\hline \hline Field & Wide 1 & Wide 2 & Wide 3 & Wide 4 \\
\hline Surface $\left(\mathrm{deg}^{2}\right)$ & 65.25 & 22.28 & 45.54 & 20.88 \\
\hline $\mathrm{B}=$ Black & 5346671 & 2100453 & 3858738 & 2040682 \\
RM = Red+magenta & 2105404 & 945090 & 1517133 & 977875 \\
M = Magenta & 98954 & 42529 & 77374 & 36181 \\
G = Green & 397921 & 135148 & 274601 & 145703 \\
\hline M/RM & 0.047 & 0.045 & 0.051 & 0.037 \\
G/RM & 0.189 & 0.143 & 0.181 & 0.149 \\
\hline
\end{tabular}

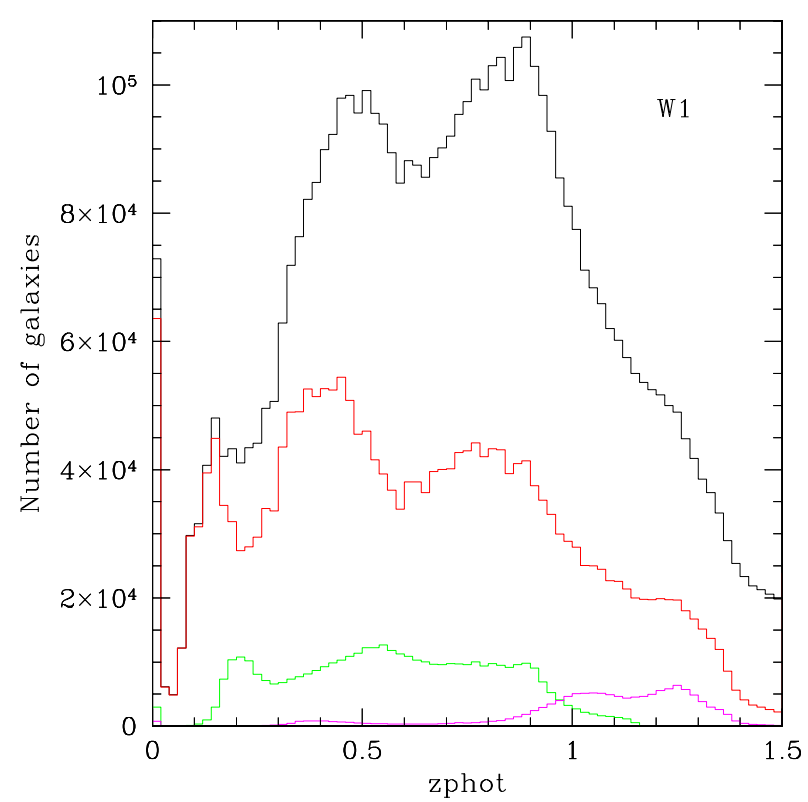

Fig. 2. Photometric redshift histograms of the galaxies in the Wide 1 field for the various magnitude selections described above. The colours of the lines are the same as the colours of the points in Fig. 1.

magnitudes $z^{\prime} \leq 22.5$ ) and the approximate total surface covered. Owing to masked areas, the effective total surface covered may be reduced by as much as $10 \%$ relatively to these values. As a comparison, the surfaces covered by the T0004 Data Release and analysed by A10 were approximately 19, 5, and $11 \mathrm{deg}^{2}$ for the W1, W3, and W4 fields respectively. W2 was not available.

To see how the different magnitude selection criteria (i.e. $z^{\prime} \leq$ 22.5 versus $y \leq 23$ ) can affect the redshift distributions of the detected clusters, we show in Fig. 2 the galaxy redshift distributions corresponding to the various magnitude selections described above for the Wide 1 field. We see that with our new magnitude selection criterium we are missing low/intermediateredshift objects but gaining high-redshift red objects. We do not show the photometric redshift histograms for the three other fields since they are similar. That three peaks are seen at redshifts of about $0.15,0.5$, and 0.9 in all four Wide fields is obviously due to degeneracies in the photometric redshift computations.

\subsection{Density maps}

For each Wide field, galaxy catalogues were built in running slices of 0.1 in redshift, shifted by 0.05 (i.e. the first slice covers redshifts 0.1 to 0.2 , the second 0.15 to 0.25 , etc.). We assumed 
the most likely photometric redshift (i.e. the one with the minimum $\chi^{2}$ value) for each object in order to assign it to a redshift slice.

As already mentioned in A10, the 0.1 redshift width of the studied slices is the best compromise between the redshift resolution and the possible dilution of the density signal due to photometric redshift uncertainties. Assuming the worst possible photometric redshift statistical error (for $z^{\prime}=22.5$, see Coupon et al. 2009) leads to a $1 \sigma$ error of 0.09 at $z=1.15$ (the upper limit in redshift for the wide field analyses).

Since the catalogues thus obtained were too large to allow direct computation of density maps, we cut them into square regions of $0.9 \times 0.9 \mathrm{deg}^{2}$, with an overlap of $0.1 \mathrm{deg}$ in both directions.

Density maps were then computed for each subcatalogue in each redshift slice, based on an adaptative kernel technique described in M07, with 1000 bootstrap resamplings of the maps to have the correct background level. The pixel size was 0.54 arcmin and the highest redshift slice was 1.05-1.15.

The SExtractor software was then applied to the galaxy density maps to detect structures at pre-defined significance levels (hereafter $S / N$ ) of $2 \sigma_{\mathrm{S}}, 3 \sigma_{\mathrm{S}}, 4 \sigma_{\mathrm{S}}, 5 \sigma_{\mathrm{S}}, 6 \sigma_{\mathrm{S}}$, and $9 \sigma_{\mathrm{S}}$ (where $\sigma_{\mathrm{S}}$ is the SExtractor detection threshold). The $9 \sigma_{\mathrm{S}}$ threshold was not considered in the A10 analysis, but we include it here to have as much information as possible on the possible presence of very massive clusters. The $2 \sigma_{\mathrm{S}}$ threshold is obviously low, but as shown in Sect. 4.2.1. we redetect almost $40 \%$ of the Thanjavur et al. (2009) clusters at $2 \sigma_{\mathrm{S}}$, so we can expect that about $40 \%$ of the clusters that we detect at a $2 \sigma_{\mathrm{S}}$ level are real.

The structures were then assembled in larger structures (called detections in the following) using a friends-of-friends algorithm (see Adami \& Mazure 1999). In A10, two detections with centres distant by less than 2.7 arcmin (the typical error on cluster positions, as estimated by A10) were merged into a single one that was assigned the redshift of the highest $S / N$ detection. Here we chose a more conservative value of $3.0 \mathrm{arcmin}$ and decided not to merge detections within 3.0 arcmin into a single one if their photometric redshifts differed by more than 0.2 . This choice was made to avoid losing clusters that could be more or less aligned along the line of sight but located at very different redshifts. The difference on the number of candidate clusters in the Wide 1 field when choosing 2.7 or 3 arcmin is $10.5 \%$.

The uncertainties on the positions of the cluster centres estimated in A10 from the comparison with the Millennium simulations were computed in the following way. We selected all the Millennium haloes present in a detection ellipse and more massive than $10^{12} M_{\odot}$. The error on the position of the ellipse centre was assumed to be the distance from the closest Millennium halo. This process naturally underestimates the uncertainty on the centre position, as the Millennium simulation always provides more than one halo more massive than $10^{12} M_{\odot}$ per detection ellipse.

For a given detection, if we had instead computed the mean difference between the ellipse centre and all the Millennium halo centres, the uncertainty on our ellipse position would have been $2.9 \pm 2.0$ arcmin, close to the predicted ellipse position uncertainty when comparing our results with other observed catalogs. We thus obtained catalogues of galaxy cluster candidates in the various CFHTLS Wide fields with a specified significance level.

\subsection{Previous detection rate assessments}

To assess our detection levels, the same method was applied to a modified version of the Millennium numerical simulation
Table 2. Relation between the SExtractor detection threshold and the minimal and mean (over all the associated Millennium haloes) cluster masses.

\begin{tabular}{lcc}
\hline \hline $\begin{array}{l}S / N \\
\left(\sigma_{\mathrm{S}}\right)\end{array}$ & $\begin{array}{c}\text { Minimal mass } \\
\left(M_{\odot}\right)\end{array}$ & $\begin{array}{c}\text { Mean mass } \\
\left(M_{\odot}\right)\end{array}$ \\
\hline 6 & $5.5 \times 10^{13}$ & $12.6 \times 10^{14}$ \\
5 & $3.5 \times 10^{13}$ & $1.3 \times 10^{14}$ \\
4 & $3.3 \times 10^{13}$ & $1.8 \times 10^{14}$ \\
3 & $1.3 \times 10^{13}$ & $1.8 \times 10^{14}$ \\
2 & $1.0 \times 10^{13}$ & $1.3 \times 10^{14}$ \\
\hline
\end{tabular}

(e.g., Springel et al. 2005, http: //www .mpa-garching.mpg . de/galform/virgo/millennium/), as described by A10. We refer to this paper (and in particular to Fig. 5 for the detection success rate and to Fig. 7 for the catalogue purity) for a full discussion, and only summarize a few points below.

For the Wide survey, only Millennium haloes more massive than $7.5 \times 10^{13} M_{\odot}$, can be detected with a success rate over $\sim 20 \%$. For lower mass systems, the detection rates become quite low at $z \geq 0.6-0.7$. False detection rates were basically found to be zero for $S / N \geq 4$ and remain small for $S / N \leq 3$ and $z \leq$ 0.8 . The typical uncertainty on the candidate cluster coordinates was typically $\sim 2.7$ arcmin (see A10, Sect. 4.2) and the redshift uncertainty was smaller than 0.2.

A minimal mass based on the photometry (and on the Millennium simulation halo masses) can be given for each cluster, assuming that the detection threshold at which the cluster is detected is a rough estimate of its richness. For each of our detections we have several Millennium haloes, and the minimal mass for this detection is taken to be that of the Millennium halo of lowest mass. The numbers were given by A10 (Table 2), but we repeat them in Table 2 for completeness.

As shown in A10, haloes with a total mass lower than $5 \times$ $10^{14} M_{\odot}$ are not strongly substructured while more massive $d e-$ tections can be strongly substructured. Because the Millennium simulation covers only an area corresponding to $1 \mathrm{deg}^{2}$, it includes no cluster corresponding to a $9 \sigma$ detection in our study, so we cannot give the corresponding masses in Table 2. Because of the mentioned limits of the Millennium simulation, we cannot estimate the level of real detections at $z>0.7$ or for masses higher than $2 \times 10^{14} M_{\odot}$.

\section{Results: spatial and redshift distributions of the detections}

\subsection{Detection counts}

The full lists of detections with their coordinates, redshift, and $S / N$ for the four CFHTLS Wide fields will be available electronically from the VizieR interface of the Simbad database ${ }^{2}$. The numbers of candidate clusters are given in Table 3 for each field and significance level. Altogether we detect 6802 candidate clusters in a total surface of about $154 \mathrm{deg}^{2}$ (at confidence levels between $2 \sigma$ and $9 \sigma$ ). The number of clusters detected per square degree is between 39 and 50. If we only take clusters detected at a significance level of at least $3 \sigma_{\mathrm{S}}$ into account, the total number becomes 4061 , and the numbers per square degree range between 21 and 28 .

${ }^{2}$ http://vizier.u-strasbg.fr/viz-bin/VizieR 
Table 3. Number $N$ of candidate clusters detected in the four Wide fields at various significance levels.

\begin{tabular}{lcccc}
\hline \hline$S / N$ & $\mathrm{~W} 1$ & $\mathrm{~W} 2$ & $\mathrm{~W} 3$ & $\mathrm{~W} 4$ \\
$\sigma_{\mathrm{S}}$ & & & & \\
\hline 9 & 70 & 24 & 71 & 16 \\
6 & 218 & 75 & 178 & 47 \\
5 & 204 & 55 & 173 & 65 \\
4 & 442 & 125 & 222 & 112 \\
3 & 901 & 230 & 441 & 211 \\
2 & 1412 & 410 & 734 & 366 \\
\hline$N\left(2-9 \sigma_{\mathrm{s}}\right)$ & 3247 & 919 & 1819 & 817 \\
$N\left(3-6 \sigma_{\mathrm{s}}\right)$ & 1835 & 509 & 1085 & 451 \\
$N\left(3-9 \sigma_{\mathrm{s}}\right)$ & 1905 & 533 & 1156 & 467 \\
\hline Surface $\mathrm{S}\left(\mathrm{deg}^{2}\right)$ & 65.25 & 22.28 & 45.54 & 20.88 \\
\hline$N\left(2 \sigma_{\mathrm{s}}-9 \sigma_{\mathrm{s}}\right) / S$ & 49.8 & 41.3 & 39.9 & 39.1 \\
$N\left(3 \sigma_{\mathrm{s}}-9 \sigma_{\mathrm{s}}\right) / S$ & 28.1 & 22.8 & 23.8 & 21.6 \\
\hline
\end{tabular}

Thanjavur et al. (2009) searched for clusters in the W1 field, based on the T0005 CFHTLS data release. They found 6144 galaxy clusters, of which 239 are rich clusters, and thus detected about 35 clusters $/ \mathrm{deg}^{2}$. This number is intermediate between the densities of clusters detected at $2 \sigma_{\mathrm{s}}$ and $3 \sigma_{\mathrm{s}}$. Since about $55 \%$ of the clusters that we have detected at $2 \sigma_{\mathrm{s}}$ are likely to be real (see Sect. 4.1), we can say that the cluster densities that we give in Table 3 agree with the number given by Thanjavur et al. (2009). These authors have also sent us their cluster catalogues in the three other Wide fields, and we will make a detailed comparison of our numbers with theirs in Sect. 4.2.1.

\subsection{Spatial distributions of the detections}

We show in Figs. 3-6 the spatial distributions of our detections in the four Wide fields (since the W1 field is larger than the other ones, Fig. 3 was divided into four redshift bins for the sake of clarity). As expected, candidate clusters are distributed more or less homogeneously in all the fields.

\subsection{Redshift distributions of the detections}

The photometric redshift distributions of our cluster detections in the four Wide fields are shown in Fig. 7, where we plot the density of clusters per square degree in photo- $z$ bins of 0.1 (between $z=0.1$ and 1.15). As expected, these numbers are quite comparable in the four fields, suggesting that there is no systematic difference, although the four Wide fields sample very different regions of the sky. If we exclude the clusters detected only at the $2 \sigma_{\mathrm{s}}$ level, the shapes of the histograms remain the same, but the numbers of detected clusters are notably reduced.

On the other hand, there seems to be a significant dependence of the number of detections at high redshift with the signal-to-noise level. This is illustrated by Fig. 8, where we see that the number of high-redshift clusters detected increases as the detection level decreases. We checked that this is consistent with cosmological predictions. Evrard et al. (2002) have computed the expected number of clusters as a function of cluster mass in different redshift intervals (see their Fig. 7, right column). For clusters of mass $10^{14} M_{\odot}$, they predict about four times more clusters in the redshift range $0.5 \leq z<1.2$ than in the range $0.2 \leq z<0.5$. This is consistent with what we find. Very massive clusters detected at the $9 \sigma_{\mathrm{S}}$ level are present in small numbers and are found mainly at redshifts lower than 0.6.

\section{Comparison with other cluster detections in the CFHTLS}

Before carrying out analyses based on our catalogue of candidate clusters, we discuss the validity of the catalogue and its limitations by comparing our detections with those of other authors. Several other cluster candidate catalogues exist in the CFHTLS areas. The T0006 release provides better photometric redshifts than previous versions, but it remains useful to compare our detections with previous catalogues. In particular, the candidate catalogues of A10 were based on the T0004 release and detection rates were assigned, as already quoted, by comparisons with the Millennium simulation. Obviously, one can only estimate the effects of the biases that were previously included in the simulations. It is therefore also useful to compare our detections with other real catalogues to uncover unexpected biaises. Moreover, the modified Millennium simulations considered in A10 used photometrically-mimicked redshifts based only on the mean CFHTLS uncertainties and not including the catastrophic failures sometimes seen in photometric redshifts. We show in the following that this sometimes has non-negligible effects.

\subsection{Internal assessment}

We cross-correlated our new catalogues of cluster candidates with those of A10, matching all candidate clusters located at distances smaller than 4.2 arcmin (quadratic sum of the typical 3 arcmin position uncertainty of our candidate clusters) and with photometric redshifts differing by less than 0.2 . In A10, the total numbers of candidate clusters were 755, 175 and 99 in the W1, W3, and W4 fields respectively; the corresponding numbers in the present work are 3247,1819 , and 817 . We remind the reader that the solid angle covered is now notably larger.

Out of the 1029 clusters detected in A10, we redetect only 588 clusters in W1, W3, and W4. To see if the non-redetections occurred in specific spatial regions, we plotted the positions of the non-redetected clusters. In all three Wide fields, the nonredetected clusters are spread over the entire regions covered by A10, so there is no spatial effect.

We also tested the hypothesis that non-redetections could come from selecting galaxies with $i^{\prime} \leq 23$ in A10 and galaxies with $z^{\prime} \leq 22.5$ in the present work could lead to different samples (the $i^{\prime}$ filter was replaced by a $y$ filter between the T0004 and T0006 data releases, as explained in Sect. 2.1). For this, we compared the mean $i^{\prime}-z^{\prime}$ and $y-z^{\prime}$ colours in the entire T0006 sample used here, in the galaxies found to belong to clusters in T0006, and in the subcatalogues of galaxies in the clusters that were detected by A10 but not redetected in the present work. The mean values are given in Table 4 for the W1, W3, and W4 fields. As seen from this table, only small differences are found, so the different selections do not seem to introduce a bias.

We also estimated the $i^{\prime}-z^{\prime}$ colours in the $i^{\prime} \leq 23$ and $z^{\prime} \leq$ 22.5 selected samples for the four Wide fields. We find $i^{\prime}-z^{\prime}$ between 0.23 and 0.26 for the $i^{\prime} \leq 23$ sample and $i^{\prime}-z^{\prime}$ between 0.32 and 0.34 in the $z^{\prime} \leq 22.5$ sample. Therefore the fact that we had to change our magnitude selection between T0004 and T0006 introduces a change in colour of $i^{\prime}-z^{\prime}$ smaller than 0.1 .

We now closely examine which T0004 candidate clusters are not redetected in the T0006 data. We first test the influence of redshift. We show in Fig. 9 the redetection percentage as a function of the T0004 candidate cluster redshifts. We see that redetection percentages are close to $70 \%$ between $z=0.375$ and 1.05. At lower redshifts, redetection percentages are very 

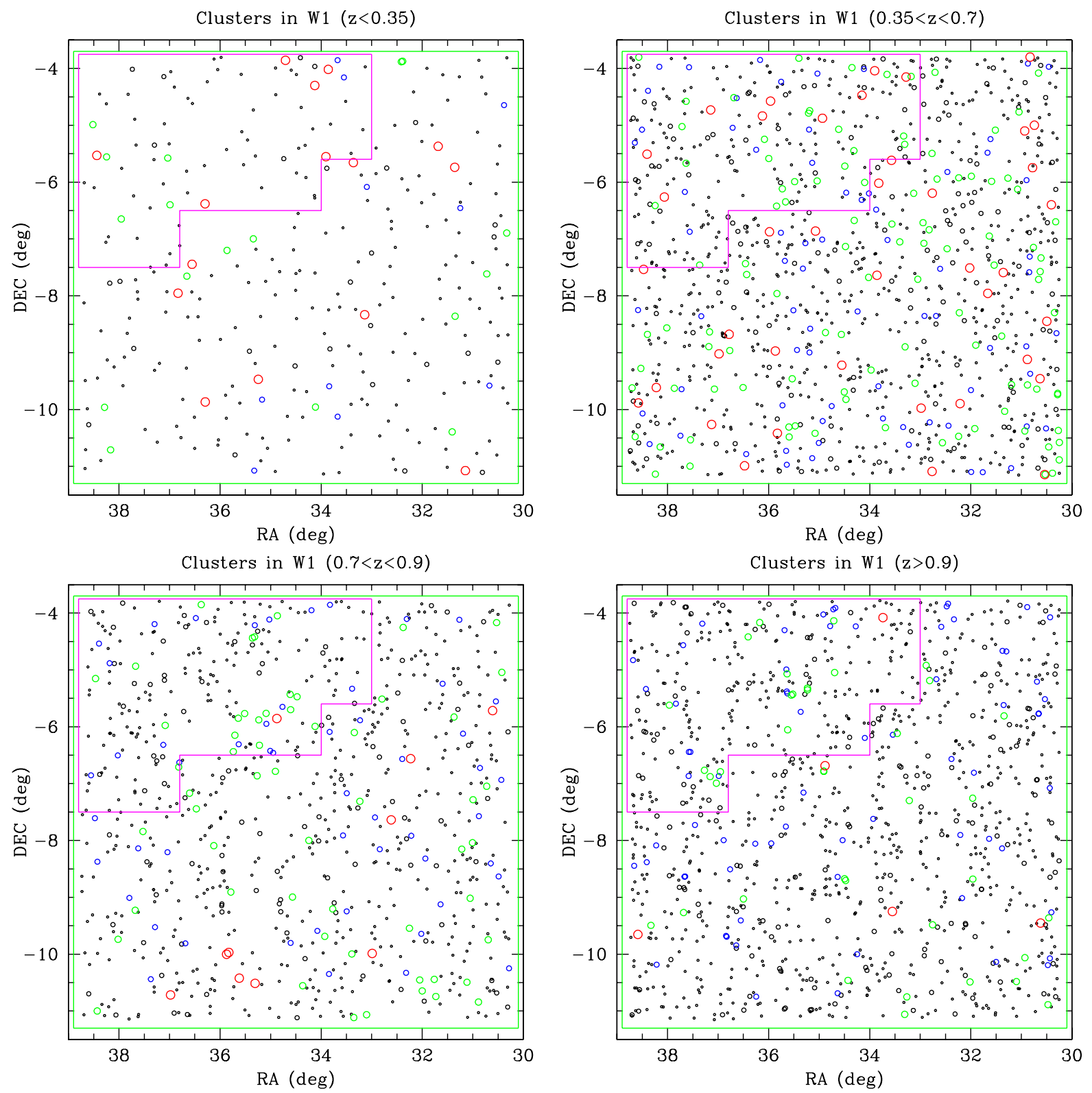

Fig. 3. Spatial distribution of our detections in the W1 field, divided in four photometric redshift bins for the sake of clarity: top left $z<0.35$; top right: $0.35<z<0.7$; bottom left $0.7<z<0.9$; bottom right: $z>0.9$. The symbols are large red circles: $S / N \geq 9$; large green circles: $S / N \geq 6$; medium blue circles: $S / N \geq 5$; medium black circles: $S / N \geq 4$; small black circles: $S / N \geq 3$ and $S / N \geq 2$. The area covered by the T0004 data release analysed by A10 is shown in magenta.

low, and not higher than the random probability (computed from the average number of clusters per unit volume) of a match between T0004 and T0006 candidate clusters (given the number of respective T0004 and T0006 cluster candidates in the considered volume) when considering redshifts lower than 0.3 . This could be explained if the $u^{*}$ CFHTLS filter was not very efficient alone in helping to locate the Balmer break when computing photometric redshifts. It is probably not only by chance that we see the redetection percentages strongly grow when the Balmer break exits the $g^{\prime}$ band, i.e. when photometric redshifts do not rely only on the $u^{*}$ band data to locate the break. We find the same behaviour at redshifts above 1.05 , where photometric redshifts only rely on the $z^{\prime}$ band to locate the Balmer break. The redshift histograms of galaxies in regions of $\mathrm{W} 1, \mathrm{~W} 3$, and $\mathrm{W} 4$ covered both by T0004 and T0006 are somewhat different, with more low-redshift galaxies in T0006 and more high-redshift galaxies in T0004. Such differences are due to the improvement in the photometric redshift determinations between T0004 and T0006. Figure 9 therefore strongly speaks in favour of only selecting candidate clusters in the $[0.375,1.05]$ redshift interval.

We now test the effect of the candidate cluster $S / N$. We show in Fig. 10 the redetection percentage as a function of the $S / N$ for clusters in the $[0.375,1.05]$ redshift interval where the redetection percentage is the highest. This figure clearly shows a regular increase in the percentage from $\sim 55 \%$ when $S / N=2$ to $\sim 80 \%$ when $S / N \geq 6$.

Low $S / N$ candidate clusters therefore seem to be only poorly redetected, especially for $S / N=2$. A possible explanation for this effect is that part of such poor structures are made of projected galaxy concentrations on the sky with artificial redshift 


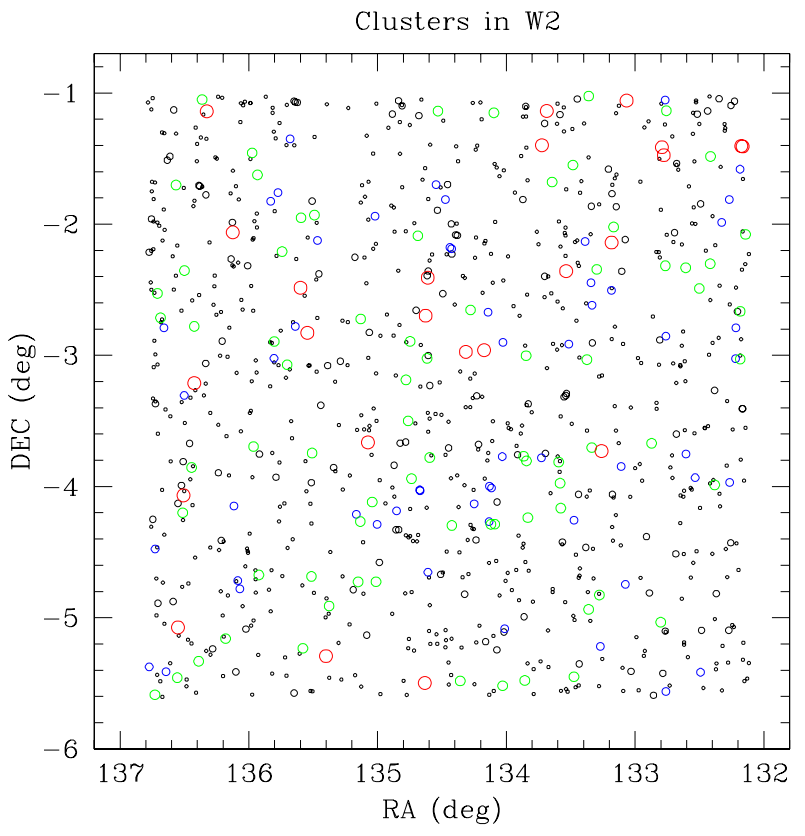

Fig. 4. Same as Fig. 3 for the W2 field. All redshifts are included.

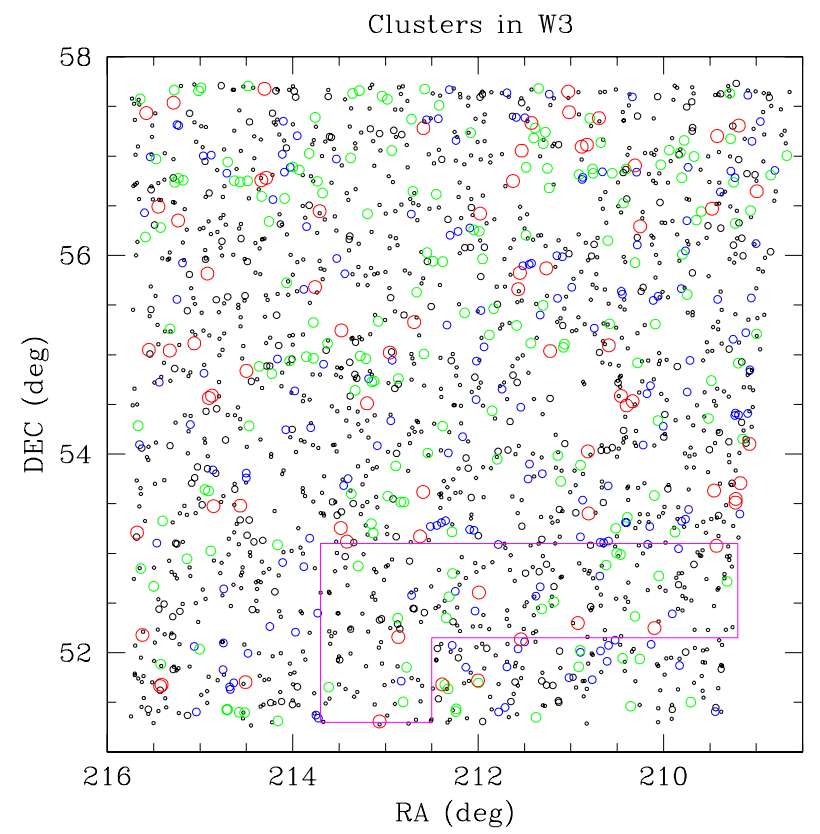

Fig. 5. Same as Fig. 4 for the W3 field. The area covered by the T0004 data release analysed by A10 is shown in magenta.

concentrations, produced by catastrophic photometric redshift failures that induce photometric redshift accumulations at given values. Such features are commonly found, for example, in Coupon et al. (2009), and are not always easily detected when only a few galaxies are involved. A way to test this hypothesis is to consider the 33 T0006 candidate clusters in the VVDS spectroscopic area. It is then possible to compute the percentage of these catastrophic photometric redshift failures in the corresponding clusters. A galaxy will be assigned to a cluster if the redshift difference between the galaxy and the cluster is smaller than 0.1 and if the galaxy is closer than $1 \mathrm{Mpc}$ (in projection,

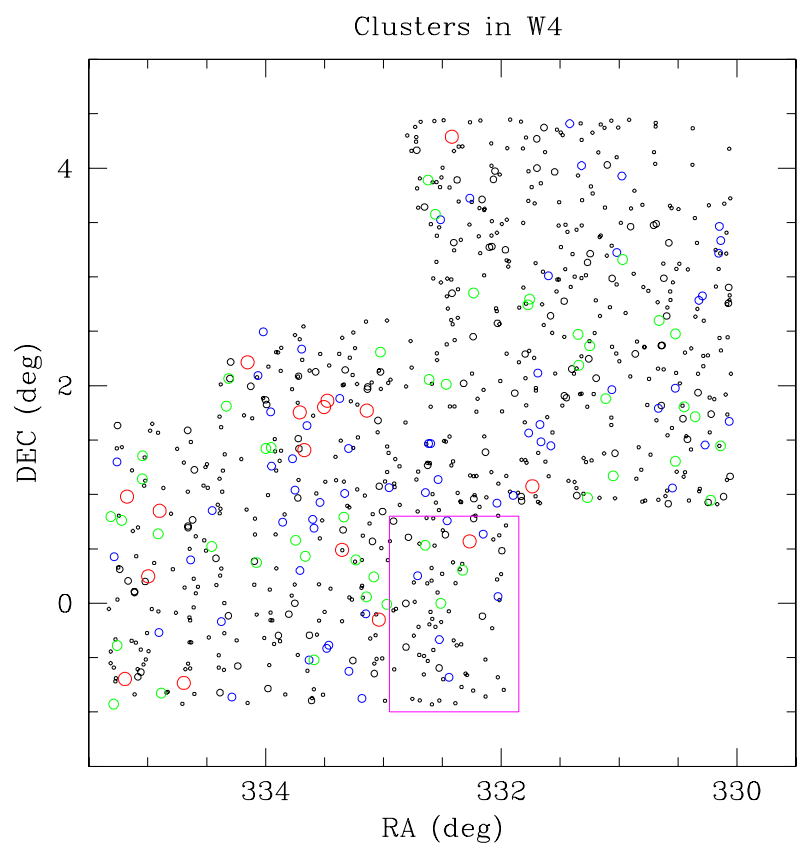

Fig. 6. Same as Fig. 4 for the W4 field. The area covered by the T0004 data release analysed by A10 is shown in magenta.

Table 4. Mean values of the mean $i^{\prime}-z^{\prime}$ and $y-z^{\prime}$ colours in the entire T0006 field, in the entire T0006 cluster sample and in the subcatalogue of galaxies in the clusters detected in T0004 and not redetected in T0006.

\begin{tabular}{ccccccc}
\hline \hline & $\begin{array}{c}\text { T0006 } \\
\text { all } \\
i^{\prime}-z^{\prime}\end{array}$ & $y-z^{\prime}$ & $\begin{array}{c}\text { T0006 } \\
\text { clusters } \\
i^{\prime}-z^{\prime}\end{array}$ & $y-z^{\prime}$ & $i^{\prime}-z^{\prime}$ & $y-z^{\prime}$ \\
\hline W1 & 0.32 & 0.34 & 0.37 & 0.38 & 0.38 & 0.35 \\
W3 & 0.32 & 0.35 & 0.37 & 0.38 & 0.35 & 0.35 \\
W4 & 0.34 & 0.43 & 0.38 & 0.46 & 0.34 & 0.46 \\
\hline
\end{tabular}

calculated at the cluster distance) from the cluster centre. The same conditions will apply for Sect. 5. We give in Fig. 11 the percentage of catastrophic photometric redshift failures in the 33 T0006 candidate clusters in the VVDS spectroscopic area as a function of the candidate cluster $S / N$. The position of the horizontal line in Fig. 11 implies that the photometric redshifts are always worse for galaxies in clusters than in the whole field, as already noted by Guennou et al. (2010). This is true in particular for cluster dominant and bright spiral galaxies, which are not well fit by the template spectra available to train photometric redshift codes. Figure 11 shows that on the one hand, we clearly see that $S / N \geq 5$ candidate clusters have a behaviour similar to that of the whole sample. On the other hand, $S / N=2$ candidate clusters exhibit catastrophic photometric redshift percentages that are nearly three times higher. However, if we compute the catastrophic photometric percentages only for those $S / N=2$ candidate clusters that were both detected in A10 with the T0004 data and with the T0006 data, the percentage value diminishes strongly. A plot of the photometric versus spectroscopic redshift for the clusters found in the XMM-LSS survey can be found in Appendix A.1 of Adami et al. (2011). 

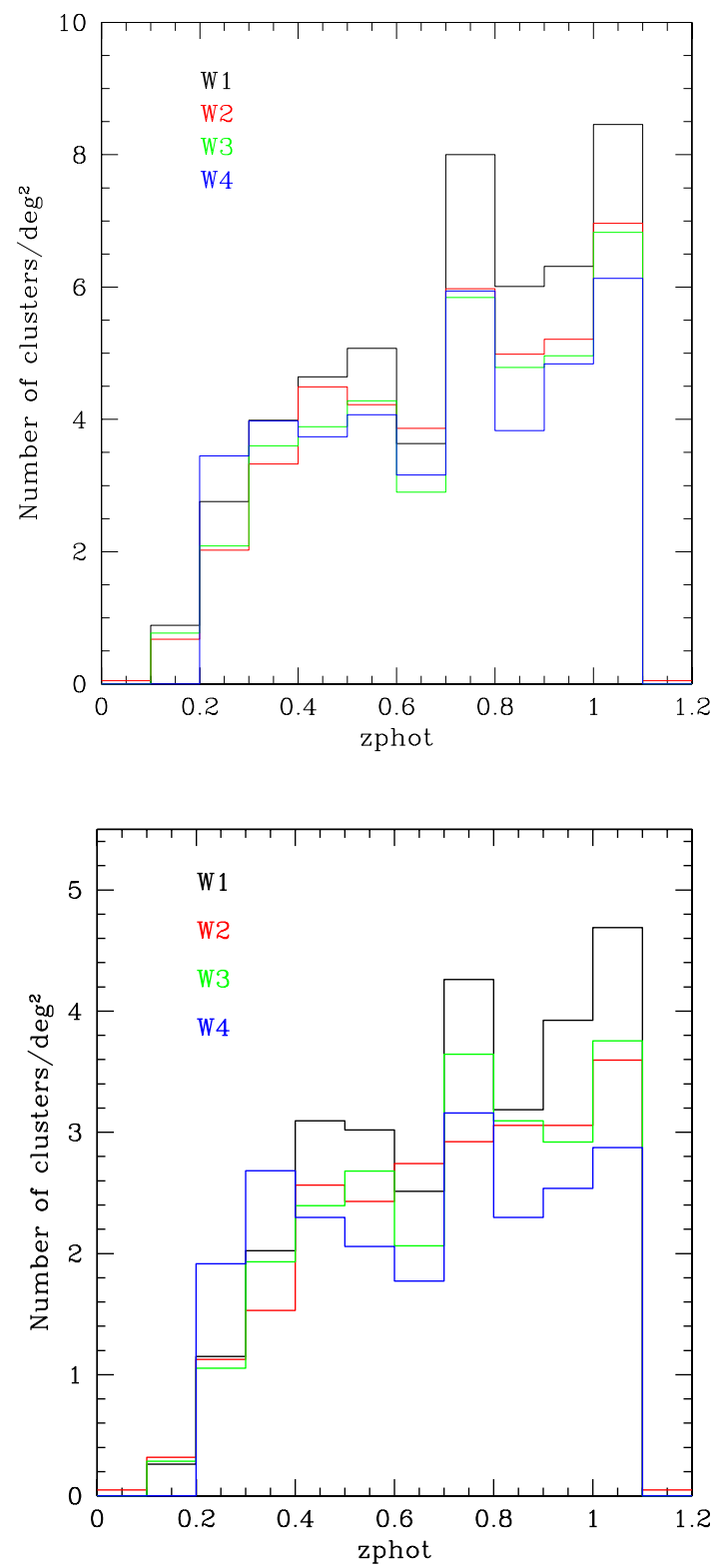

Fig. 7. Histograms of the surface density of clusters in photometric redshift bins of 0.1 for all the candidate clusters in the four CFHTLS-Wide fields (top) and for all the candidates detected at $3 \sigma_{\mathrm{s}}$ or above (bottom).

We therefore conclude that $S / N=2$ candidate clusters detected in A10 and not redetected with T0006 data are at least partly not real. They are probably made of artificial redshift concentrations on the sky produced by catastrophic photometric redshift failures. This also explains why the $S / N=2$ candidate cluster percentage in Fig. 10 (typically 50\%) is lower than the predicted values in A10 $(\sim 80 \%)$. This is because catastrophic photometric redshift failures were not taken into account when mimicking CFHTLS photometric redshifts with the Millennium simulation redshifts. The same applies to the T0006 sample, but to a lesser extent.

As a conclusion, we therefore typically redetect $75 \%$ of the A10 candidate clusters when considering $S / N \geq 3$ structures in the $[0.375,1.05]$ redshift interval. $S / N=2$ candidate clusters are also probably mainly real when detected by both A10 and the present paper.
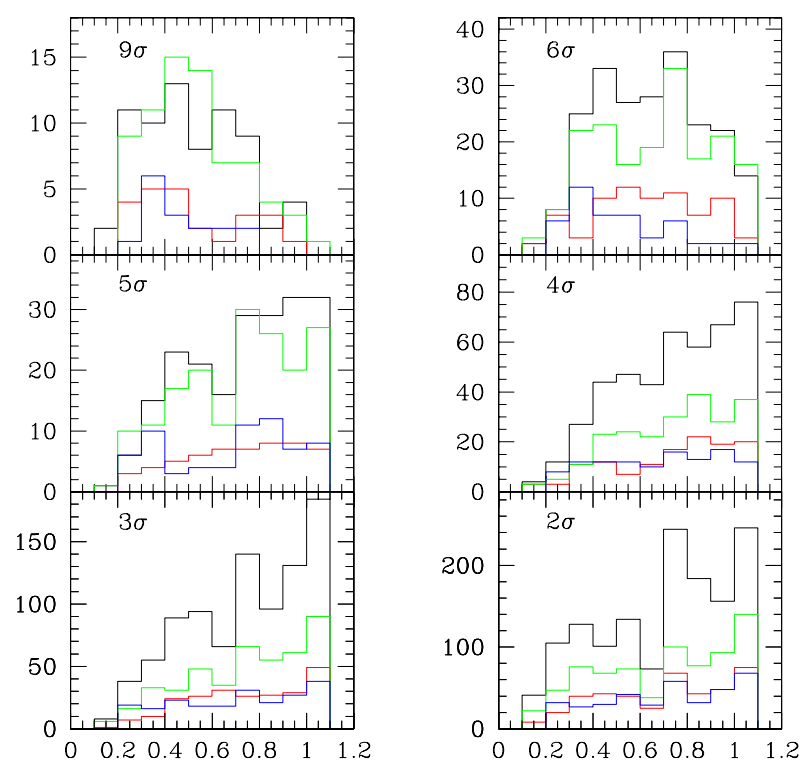

Fig. 8. Histograms of the photometric redshift distributions for the candidate clusters in the four fields, colour coded as: black for Wide 1, red for Wide 2, green for Wide 3 and blue for Wide 4 . The six figures correspond to the detection levels adopted: $9 \sigma_{\mathrm{s}}$ (top left), $6 \sigma_{\mathrm{s}}$ (top right), $5 \sigma_{\mathrm{s}}$ (middle left), $4 \sigma_{\mathrm{s}}$ (middle right), $3 \sigma_{\mathrm{s}}$ (bottom left), and $2 \sigma_{\mathrm{s}}$ (bottom right).

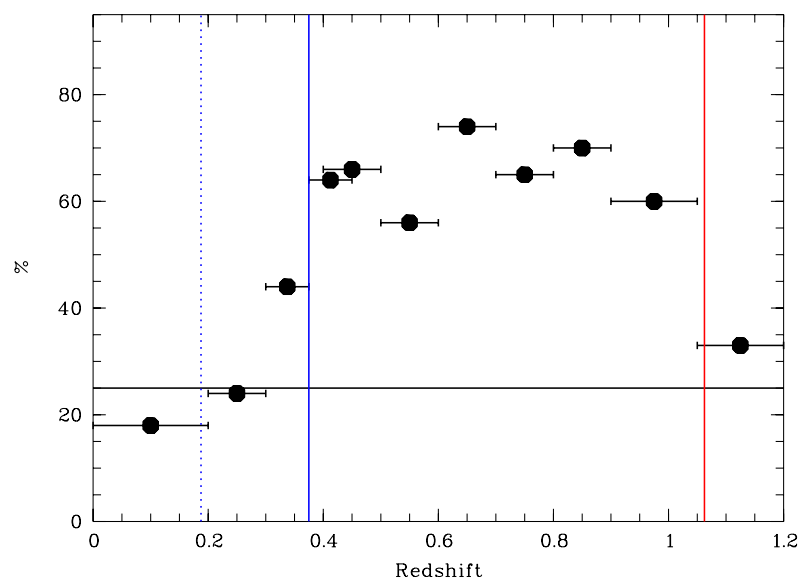

Fig. 9. Percentage of A10 candidate cluster redetection as a function of redshift. Horizontal line: statistical random probability to have a match between T0004 and T0006 candidate clusters. Vertical blue dotted line: redshift where the Balmer break passes the middle of the $g^{\prime}$ band. Vertical blue continuous line: redshift where the Balmer break exits the $g^{\prime}$ band. Vertical red line: redshift where the Balmer break enters the $z^{\prime}$ band.

\subsection{Comparison with detections in the Wide fields by other authors}

\subsubsection{Comparison with the Thanjavur et al. (2009) detections}

We correlated our catalogues of cluster candidates with those of Thanjavur et al. (2009) in the four Wide fields. The Thanjavur catalogues are based on the T0005 CFHTLS data release (which covers the same field as the T0006 release considered here). They include 2491, 1002, 1697 and 614 galaxy clusters in the $\mathrm{W} 1, \mathrm{~W} 2, \mathrm{~W} 3$, and $\mathrm{W} 4$ fields, respectively (the respective numbers of clusters in our catalogues are 3247, 919, 1819, and 817). 
F. Durret et al.: Galaxy cluster searches in the CFHTLS Wide fields

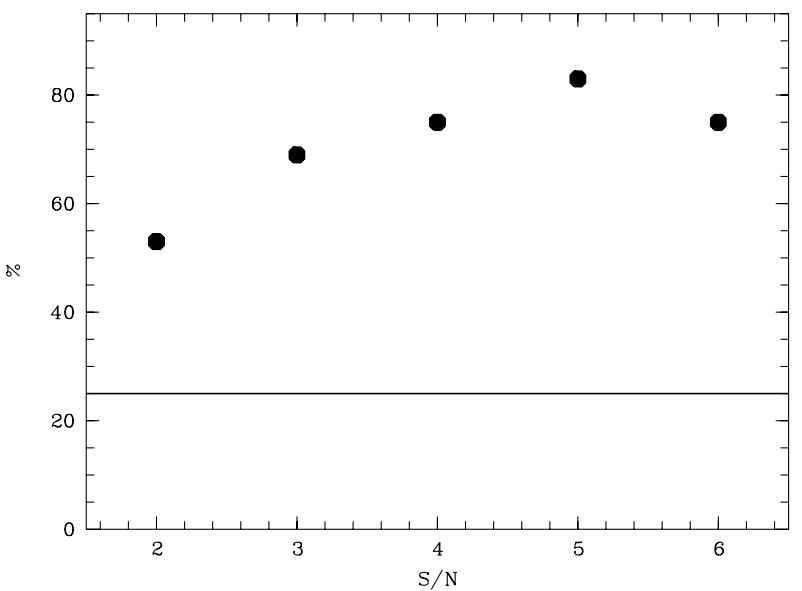

Fig. 10. Percentage of redetections of A10 candidate clusters in the $[0.375,1.05]$ redshift interval as a function of candidate cluster $S / N$. The horizontal line is the statistical random probability of having a match between T0004 and T0006 candidate clusters.

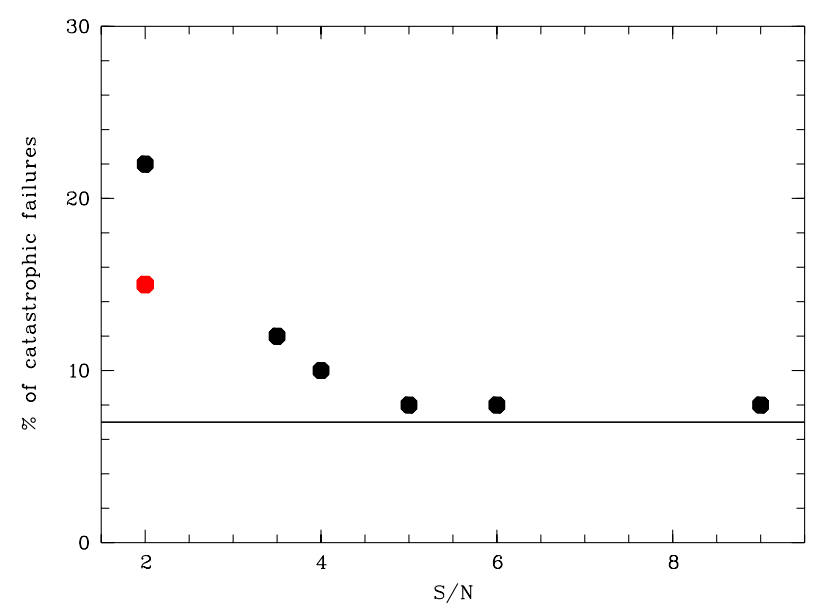

Fig. 11. Black dots: percentage of catastrophic photometric redshift failures in the 33 T0006 candidate clusters in the VVDS spectroscopic area as a function of $S / N$. The point at $S / N=3.5$ was obtained by combining the $S / N=3$ and $S / N=4$ data. Red dot: percentage of catastrophic photometric redshift failures in the $S / N=2$ T0006 candidate clusters in the VVDS spectroscopic area also detected by A10. The horizontal line shows the mean percentage of catastrophic photometric redshift failures for the whole photometric redshift sample (not only in clusters) in the VVDS area.

We made the same kind of matching as in the previous section, comparing this time our T0006 cluster candidates with the Thanjavur catalogues.

We first tested the redshift influence. Figure 12 shows the redetection percentage as a function of redshift. We see that redetection percentages are close to $55 \%$ between $z=0.375$ and 0.5 . At lower and higher redshifts, redetection percentages are lower. They are not higher than the random probability of a match for redshifts lower than 0.2 and higher than 0.7. On the low-redshift side, the explanation given in the previous section also applies: the $u^{*}$ band alone is probably not sufficient to locate the Balmer break when computing photometric redshifts. On the high-redshift side, we can note that the Thanjavur method is mostly efficient at finding clusters at redshifts lower than 0.5 (see Fig. 13). At higher redshifts, Thanjavur et al. (2009) detect less than $90 \%$ of minor clusters such as Fornax (see their Fig. 3).

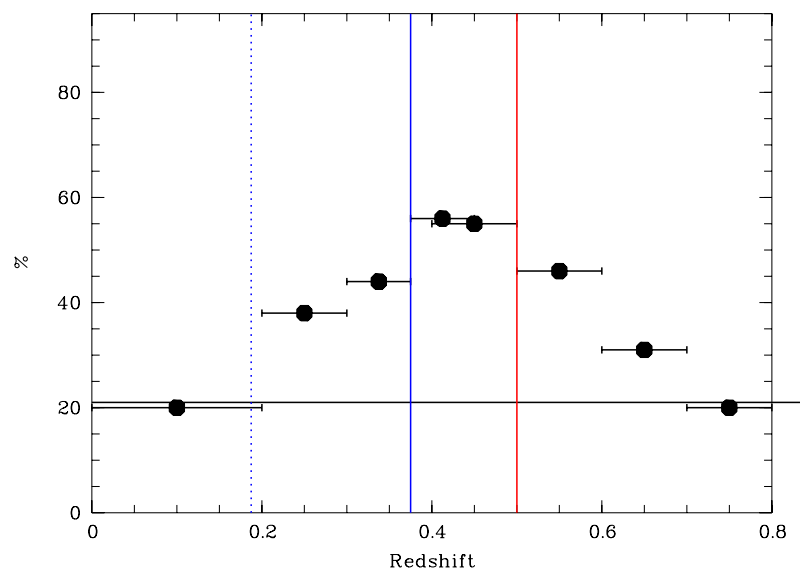

Fig. 12. Percentage of Thanjavur et al. (2009) candidate clusters redetected in the present paper as a function of redshift. Horizontal line: statistical random probability of a match. Vertical blue dotted line: redshift where the Balmer break passes the middle of the $g^{\prime}$ band. Vertical blue continuous line: redshift where the Balmer break exits the $g^{\prime}$ band. Vertical red line: redshift where the Thanjavur et al. (2009) method starts missing more than $10 \%$ Fornax-like clusters.
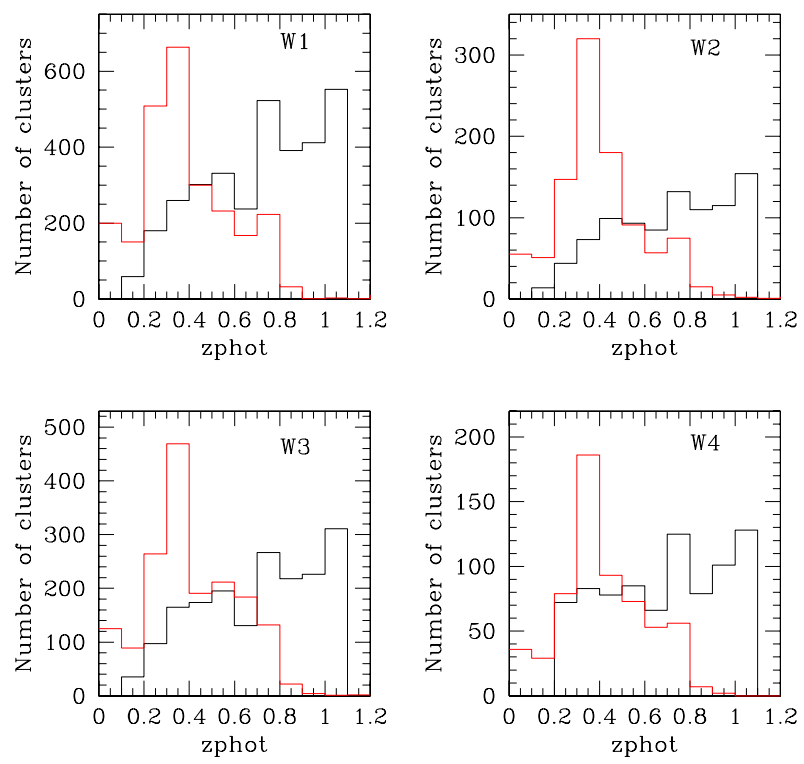

Fig. 13. Histograms of the photometric redshift distributions for the candidate clusters detected in the four Wide fields; black: our detections, red: detections by Thanjavur et al. (2009).

We now test the effect of the candidate cluster $S / N$ on the redetection of the Thanjavur et al. (2009) clusters in the $[0.375,0.5]$ redshift interval. We show in Fig. 14 the redetection percentage as a function of the $S / N$. This figure clearly shows a regular increase in the percentage from $\sim 35 \%$ when $S / N=2$ to $\sim 90 \%$ when $S / N \geq 6$. With a stricter condition that the cluster positions match within 3 arcmin, as taken in Sect. 5, the percentage increases from $\sim 25 \%$ when $S / N=2$ to $\sim 75 \%$ when $S / N \geq 6$.

These results are consistent with the conclusions of the previous section.

\subsubsection{Comparison with the Limousin et al. (2009) detections}

Limousin et al. (2009) did a search for (massive) galaxy groups (i.e. with masses between $10^{13}$ and $10^{14} M_{\odot}$ ) in a large area of 


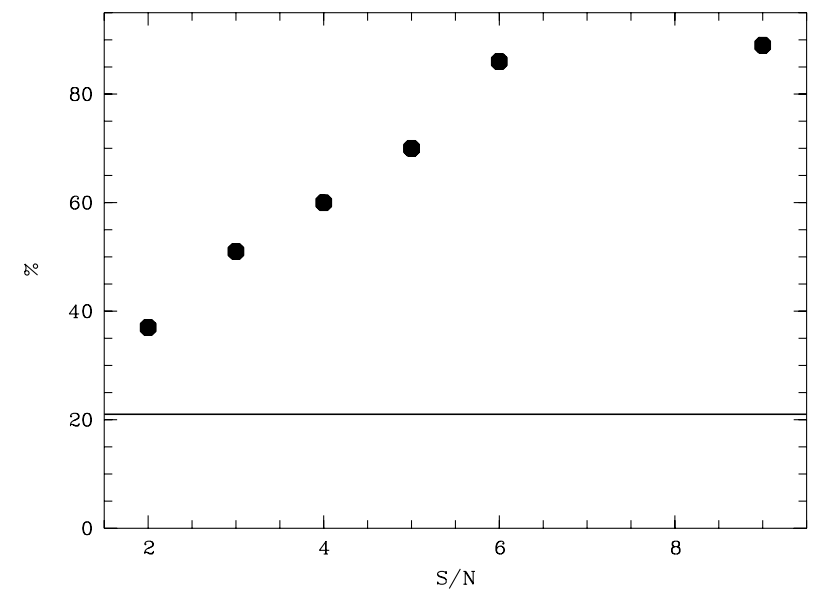

Fig. 14. Percentage of redetections of Thanjavur et al. (2009) candidate clusters in the $[0.375,0.5]$ redshift interval as a function of the candidate cluster $S / N$. The horizontal line is the statistical random probability of a match.

$100 \mathrm{deg}^{2}$ and detected 13 objects in the Wide fields. These objects are not easy targets for our method, since they are only groups, therefore not very rich in terms of galaxies. However, we redetect seven of these 13 groups in our data. Among the six that are not redetected, three are located in CFHTLS masked regions in at least one band and therefore are not detectable by our method by definition. This is because we rely on galaxy detections in the cluster, while lensing methods are based on peripherical objects and can detect a cluster even if it is partly in a masked region. Another cluster is only detected by one method in Limousin et al. (2009) (at the edge of a field) and is therefore not very secure. Finally, the last one is the optically poorest group in Limousin et al. (2009), so the hardest to detect with our method. We therefore conclude that we detect between 80 and $90 \%$ of the Limousin et al. (2009) groups accessible by our method.

\subsection{Comparison with detections in the Deep fields}

Obviously, the shallowness of the Wide fields relative to the Deep fields implies that the numbers of clusters detected in the Wide fields will be smaller. We have shown in A10 that the ratio of the numbers of detected clusters in the Deep and Wide fields is expected to be $2.7 \pm 1.4$. However, we expect to redetect at least a fraction of the clusters with redshifts $z \leq 1.15$ found in the Deep fields.

\subsubsection{Comparison with the Olsen et al. (2007) and Grove et al. (2009) detections}

Olsen et al. (2007) detect 162 clusters over an area of $3.112 \mathrm{deg}^{2}$ in the CFHTLS Deep 1-3, and 4 fields applying the matched filter method. Out of these, 46 were in Deep 1 and 40 in Deep 3 (Deep 2 and Deep 4 are not included in W2 and W4, so we cannot try to redetect their clusters in these fields). We redetect by our method 20 of their clusters in W1 and 10 in W3.

Based on the same method, Grove et al. (2009) found 114 clusters, out of which 34 in the Deep 1 and 27 in the Deep 3 field. We redetect 20 and 12 clusters. In several cases, we redetect more clusters than the numbers found by the matched filter technique. This can be explained if we assume that we detect many low-mass structures that are still in the course of their merging process and that will produce a very massive cluster in the future. In this case, our method will detect several structures associated with a single candidate cluster found by the matched filter technique.

The ratio between the considered literature detections and our redetections is 2.3 (D1) and 4 (D3) for Olsen et al. (2007), and 1.7 (D1) and 2.25 (D3) for Grove et al. (2009). All these numbers are within the expected ratio of $2.7 \pm 1.4$.

\subsubsection{Comparison with the XMM-LSS detections}

The most recent cluster catalogue detected by the XMM-LSS was recently published by Adami et al. (2011), where they present 66 spectroscopically confirmed clusters with redshifts $0.05 \leq z \leq 1.15$ within an area of $6 \mathrm{deg}^{2}$. Their clusters were divided into four categories: $\mathrm{C} 0$ are clusters detected at optical wavelengths but not in X-rays, $\mathrm{C} 1$ are clusters with the highest probability of being real clusters, and $\mathrm{C} 2$ and $\mathrm{C} 3$ are fainter clusters with a decreasing probability of being truly extended galaxy structures, but which have passed all the spectroscopic tests.

The number of clusters in the $\mathrm{C} 1, \mathrm{C} 2$, and $\mathrm{C} 3$ catalogues of Adami et al. (2011) and in the area covered by W1 is 40. By cross correlating our catalogues with these three catalogues, we redetect 14 clusters. Among the 26 non redetected clusters, 19 are in masked CFHTLS regions so not detectable by our method. Therefore only 7 are not detected by our method and should have been. This is understandable since they are all at redshifts lower than 0.35 , where we have shown that our method is not very efficient. This represents a $65 \%$ level detection.

Our detection level depends on the category, but only weakly, and with a large dispersion: the detection levels found for the C1, C2, and C3 categories for the Adami et al. (2011) clusters are $6.0 \pm 3.6,4.3 \pm 2.1$, and $3.8 \pm 2.7$, respectively.

\subsubsection{Comparison with the Cabanac et al. (2007), Gavazzi \& Soucail (2007) and Bielby et al. (2010) detections}

Cabanac et al. (2007) detect 42 clusters with a secure redshift in the four CFHTLS Deep fields, out of which 32 in zones covered by the Wide fields that we have analysed. We redetect 13 clusters, while 14 of the non redetected clusters are in masked areas. The percentage of redetection is therefore $68 \%$.

Gavazzi \& Soucail (2007) detect 14 clusters in the Deep fields, out of which ten are in the Wide fields. Five are in masked areas and we redetect two of the remaining five. The last three non-redetected clusters are all at redshifts lower than 0.16 , where our method is not efficient.

Bielby et al. (2010) searched for clusters with redshifts higher than 1.1 in the Deep 1 and Deep 4 fields. The only cluster at redshift low enough that we may hope to redetect it is the one at 1.07 , but it is in a partially masked CFHTLS region, which definitively prevents any detection by our method.

In conclusion, our catalogue of candidate clusters is reliable with the following limitations: our most reliable detections are between redshifts 0.375 and 1.05 and for signal to noise $\geq 3$. We now proceed with a discussion of results derived from our catalogue.

\section{Preliminary analysis of a subsample of stacked clusters}

As a first test to see how the general properties of the clusters that we detect match well-known cluster properties, we considered a 
F. Durret et al.: Galaxy cluster searches in the CFHTLS Wide fields

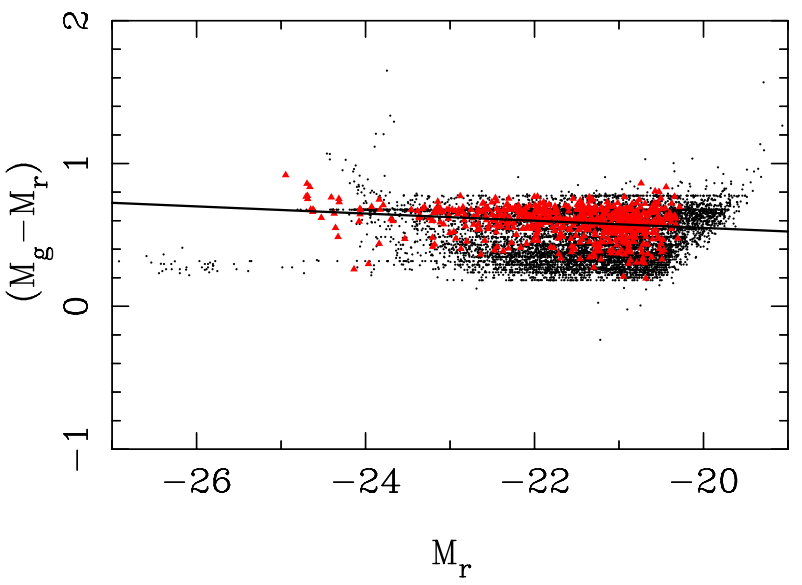

Fig. 15. Colour-magnitude diagram for 45 stacked clusters (see text). The black points are all the galaxies in a radius of $1 \mathrm{Mpc}$ around each cluster centre, and with an absolute magnitude $M_{z^{\prime}}<-21.0$. The red points correspond to the subsample of these galaxies within \pm 0.1 of the cluster redshift. The black line shows the best fit to the colour-magnitude relation drawn from the red points

subsample of 45 clusters in the W1 field detected simultaneously by A10, by the present analysis at a significance level of at least $3 \sigma_{\mathrm{s}}$ and by Thanjavur et al. (2009). We considered the absolute magnitudes calculated by Le Phare in order to be able to stack these clusters, which have redshifts of at most 0.8 . For this redshift, the limiting value $z^{\prime}=22.5$ chosen for the present analysis corresponds to an absolute magnitude $M_{z^{\prime}}=-21.0$. We therefore applied this absolute magnitude cut to the galaxy catalogue (instead of the previous magnitude cut at $z^{\prime}=22.5$ ), in order to make a galaxy selection that is independent of redshift. In doing so, we neglected the $k$-correction. Chilingarian et al. (2010) estimate $k$-corrections for galaxies of various types between redshifts 0 and 0.5 and an application is available at http: // kcor . sai.msu.ru/. A colour is requested as input, so we calculated the mean value of $r^{\prime}-z^{\prime}$ for all the galaxies of the W1 field with redshift $0.7<z<0.9$ and find $\left\langle r^{\prime}-z^{\prime}\right\rangle=0.74$. With this value of $r^{\prime}-z^{\prime}$, their script gives a $k$-correction $k_{z^{\prime}}=0.08$ in the $z^{\prime}$ band. Since this value is small and quite uncertain, we have decided to apply no $k$-correction. We then extracted for each cluster the corresponding galaxy catalogue within a physical radius of $1 \mathrm{Mpc}$ (projected distance at the cluster redshift); this radius was computed from the photo- $z$ using the cosmology calculator by Wright $(2006)^{3}$. Galaxies were considered as cluster members if their photo- $z$ was within \pm 0.1 of that of the cluster.

The colour-magnitude diagram for the 45 stacked clusters is shown in Fig. 15. The galaxies considered as belonging cluster members (the red points in Fig. 15) define a thinner colour-magnitude relation than that obtained with all the galaxies located within a projected distance of $1 \mathrm{Mpc}$ (the black points in Fig. 15), as expected if these are indeed real clusters. The linear features appearing at bright magnitudes are due to saturated objects. The best fit to the colour-magnitude relation of cluster galaxies is:

$\left(M_{g^{\prime}}-M_{r^{\prime}}\right)=(-0.046 \pm 0.099)-(0.025 \pm 0.005) \times M_{r^{\prime}}$.

We have quantified the "thinness" of the colour-magnitude relation shown in Fig. 15 by estimating the mean, median and standard deviation of the values of $\left(M_{g^{\prime}}-M_{r^{\prime}}\right)$ for the black and

\footnotetext{
3 http://nedwww.ipac.caltech.edu/
}

Table 5. Mean values obtained for the colour-magnitude relation for cluster and non-cluster galaxies.

\begin{tabular}{lcccc}
\hline \hline $\begin{array}{l}\text { Cluster galaxies } \\
M_{r^{\prime}}\end{array}$ & $\begin{array}{c}M_{g^{\prime}}-M_{r^{\prime}} \\
\text { mean }\end{array}$ & $\begin{array}{c}M_{g^{\prime}}-M_{r^{\prime}} \\
\text { median }\end{array}$ & $\begin{array}{c}\text { Nb. of } \\
\text { galaxies }\end{array}$ & st. dev. \\
\hline-20.5 & 0.57 & 0.59 & 178 & 0.12 \\
-21.5 & 0.58 & 0.60 & 235 & 0.10 \\
-22.5 & 0.61 & 0.63 & 113 & 0.09 \\
-23.5 & 0.61 & 0.64 & 38 & 0.10 \\
-24.5 & 0.68 & 0.68 & 22 & 0.14 \\
\hline Non-cluster galaxies & & & & \\
\hline-20.5 & 0.51 & 0.53 & 3127 & 0.17 \\
-21.5 & 0.47 & 0.44 & 3176 & 0.16 \\
-22.5 & 0.49 & 0.48 & 1542 & 0.16 \\
-23.5 & 0.59 & 0.65 & 478 & 0.16 \\
-24.5 & 0.68 & 0.68 & 81 & 0.19 \\
\hline
\end{tabular}

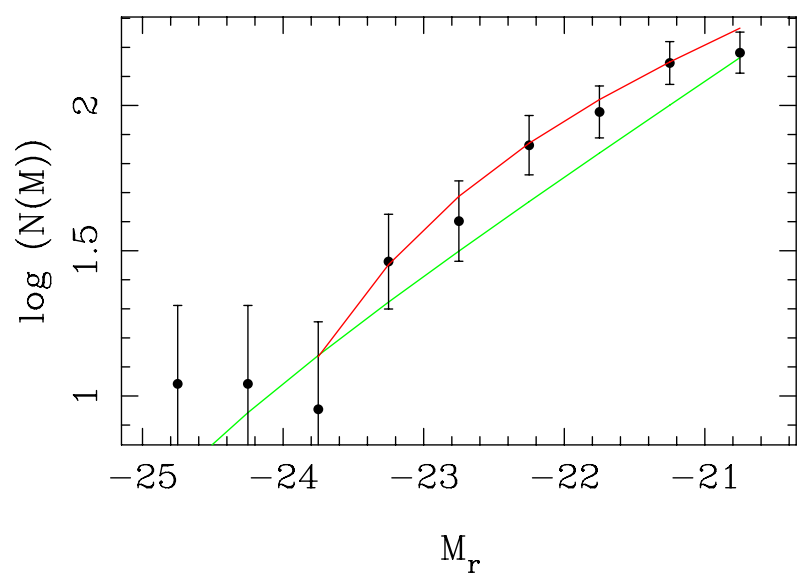

Fig. 16. Galaxy luminosity function for the galaxies of the 45 stacked clusters selected in red in Fig. 15 (showing the logarithm of the number of galaxies expressed in units of galaxies $/ \mathrm{deg}^{2} / 0.5 \mathrm{mag}$ ). The green line shows the best Schechter function fit when all the points are included and the red line shows the best Schechter function after excluding the two brightest points.

red points of Fig. 15 in $M_{r^{\prime}}$ magnitude bins of 1 . We give these values in Table 5. In all cases, the dispersions on the values of $\left(M_{g^{\prime}}-M_{r^{\prime}}\right)$ are smaller for cluster than for non-cluster galaxies, but the differences are too small to be statistically significant.

The same galaxies have been used to derive the stacked galaxy luminosity function (GLF) in the $r^{\prime}$ band. This GLF is drawn in Fig. 16, together with the best fit by a Schechter function:

$S(M)=0.4 \ln 10 \phi^{*} y^{\alpha+1} \mathrm{e}^{-y}$

where $y=10^{0.4\left(M^{*}-M\right)}$. We see from Fig. 16 that a Schechter function cannot fit the entire GLF, because there is an excess of galaxies, a feature that is not unusual in clusters (see e.g., Durret et al. 2011, and references therein). The best-fit parameters for the overall fit are $\alpha=-1.90 \pm 0.06, M_{r^{\prime}}^{*}-25.9 \pm 0.4$, and $\phi^{*}=4.0 \pm 1.6$ (galaxies $\left./ \mathrm{deg}^{2} / 0.5 \mathrm{mag}\right)$, but this is obviously a bad fit, and the value found for $M_{r^{\prime}}^{*}$ is unrealistically bright. If we exclude the two brightest points from the fit, the best-fit parameters become $\alpha=-1.47 \pm 0.23, M_{r^{\prime}}^{*}-23.44 \pm 0.47, \phi^{*}=76 \pm$ 48 , and the fit is notably better. The faint-end slope is within the usual range for clusters, i.e. between $\alpha=-1$ and -2 (see e.g., a summary of values of $\alpha$ from the literature given by Boué et al. 2008 in their Table A.1). 
A full investigation of the properties of the cluster candidates will be presented in a future paper.

\section{Angular correlation functions}

As originally shown by Bahcall \& Soneira (1983) and Klypin \& Kopylov (1983), the correlation function $\xi_{\mathrm{cc}}(r)$ of galaxy clusters can be described by a power law:

$\xi_{\mathrm{cc}}\left(r / r_{0}\right)^{-\gamma}$

where $\gamma \sim 1.8$, i.e. comparable to the correlation function of galaxies but with a significantly larger amplitude, depending also on the richness of the system.

From (1) it follows that the angular correlation function is

$\omega_{\mathrm{cc}}=\left(\theta / \theta_{0}\right)^{-\delta}$

with $\delta=\gamma-1 \sim 0.8$.

Here we performed a first test to check that the angular correlation function $w(\theta)$ of the candidate clusters in the Wide fields is consistent with what has been measured in local cluster samples. We assumed that masks are random and do not bias the cluster projected distribution. We checked that the best compromise is obtained by selecting clusters in the redshift range $0.3 \leq z \leq 0.9$ and cutting at $S / N \geq 5$ or $S / N \geq 6$. With $S / N \geq 5$ the numbers of clusters in W1, W2, and W3 are respectively 280, 89, and 229, while with $S / N \geq 6$ they are 180,61, and 142. With a lower $S / N$ cut, we would have more objects, but we would include a higher fraction of spurious clusters, poor clusters, and more distant clusters, all effects that contribute to a lower signal.

To maximize the signal, we chose a large bin in angular separation, $\log (\Delta \theta)=0.15$, and present here results obtained by selecting all candidate clusters with $S / N \geq 5$ in the redshift range $0.3 \leq z \leq 0.9$. We used the Hamilton estimator (Hamilton 1993), which is the best estimator in terms of variance, equivalent to the Landy-Szalay estimator (Landy \& Szalay 1993; see Labatie et al. 2011):

$\omega(\theta)=\frac{\operatorname{DD}(\theta) \operatorname{RR}(\theta)}{\operatorname{DR}^{2}(\theta)}-1$

where DD, RR, and DR are the number of cluster-cluster, random-random, and cluster-random pairs respectively, with angular separation in the angular bin centred on $\theta$. Our results are shown in Fig. 17, where as a reference we plot a solid line with the standard power law $\omega(\theta)=(\theta / 0.01)^{-0.8}$, and points have Poissonian error bars.

We also estimated the area-averaged correlation function $\bar{\xi}_{2}$ from counts in circular cells (see e.g., Cappi \& Maurogordato 1995). Our results are shown in Fig. 18, where errors were derived from bootstrap resamplings (which underestimate true errors). As an integral measurement, the area-averaged correlation function is less noisy than the direct two-point correlation function. The data are consistent with the expected power-law within the large error bars, and are in rough agreement with previous works on other samples (included Adami et al. 2010). A better estimate will require a selection based on cluster richness and a spectroscopic confirmation of the candidate clusters.

\section{Large-scale structures around our candidate clusters}

\subsection{Subsample}

The purpose of this section is to broaden the study initiated in Adami et al. (2011), taking advantage of the very large sample
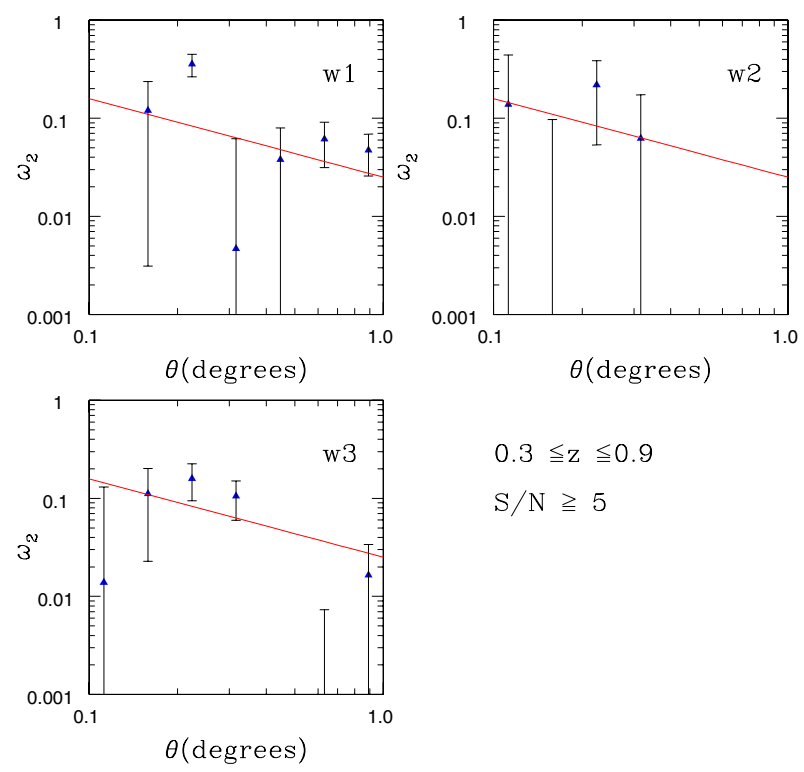

$0.3 \leqq z \leqq 0.9$

$\mathrm{S} / \mathrm{N} \geqq 5$

Fig. 17. Angular correlation functions for $\mathrm{W} 1, \mathrm{~W} 2$, and $\mathrm{W} 3$. The reference solid line has a slope of -0.8 and $\theta_{0}=0.01$ (see text).
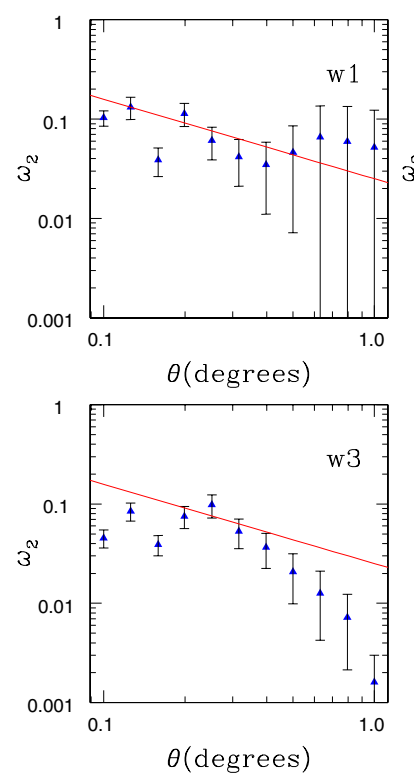

Fig. 18. Area-averaged correlation functions for $W 1$, W2, and W3. The samples include clusters with $S / N \geq 5$ and $0.3 \leq z \leq 0.9$. The reference solid line has a slope of -0.8 and $\theta_{0}=0.01$.

presently available. However, we cannot use the full sample for several reasons. First, we demonstrated in Adami et al. (2010) that the CFHTLS Wide samples are not well suited to detecting large-scale structures (LSS) as cosmological filaments at $z \geq 0.5$, so we limit the sample here to $z \leq 0.5$ candidate clusters. Second, we need a precise cluster centre localization in order not to bias the surrounding LSS detection. The uncertainty on the positions of our cluster centres is too high for our purposes, so we limit our sample to clusters also detected by Thanjavur et al. (2009) in the W1 field (the field with the broadest spatial coverage). Even though clusters detected by two different methods have a high probability of being real, the Thanjavur et al. (2009) method provides by definition a good position of the cluster centre, since it is based on the localization of the cluster $\mathrm{cD}$. We present below the LSS analysis of a sample of 491 clusters. 


\subsection{Method}

The LSS detection method is close to the one employed in Adami et al. (2011). Briefly, for a given cluster, we first selected all galaxies (in a given radius) in a \pm 0.05 redshift slice around the cluster redshift. We then counted galaxies in given magnitude intervals and in 72 angular sectors (5 deg wide and within the considered radius) around the cluster.

We selected four radii around the clusters. The first one was a $1 \mathrm{Mpc}$ radius, to consider only the densest cluster areas. The second one was a $2.5 \mathrm{Mpc}$ radius, to consider areas of the order of the virial radius. The third one was a corona between 1.5 and $3.5 \mathrm{Mpc}$ to consider only galaxy populations close to the virial radius, so just starting to experience the cluster influence. The last one was a corona between 2.5 and $5 \mathrm{Mpc}$ to consider galaxy populations not yet influenced by the considered cluster.

We selected two magnitude intervals. The first one (magnitude interval I hereafter) was the full available CFHTLS Wide sample interval $\left(z^{\prime} \leq 22.5\right)$ diminished by the distance modulus difference between a $z=0.5$ cluster and the considered cluster, corresponding to the absolute magnitude selection $M_{z^{\prime}}<-19.75$, chosen to sample the same absolute magnitude interval for each cluster of the sample. The second interval we chose (hereafter interval II) was obtained by including all galaxies brighter than the $\mathrm{cD}$ galaxy of the cluster +0.5 , or $M_{z^{\prime}}<M_{z^{\prime}, \mathrm{cD}}+0.5$, to consider only bright galaxies, and therefore to remove from the detections the LSS consisting only of faint galaxies. Such faint galaxy populated LSS were, for example, detected in Adami et al. (2009) around the Coma cluster.

The final step consisted in detecting the significant peaks (assumed to be LSS or other galaxy groups/clusters) in plots showing the numbers of galaxies versus the sector angle (see Fig. 19). In Adami et al. (2011), we chose to consider a peak as significant if it was at more than three times the $1 \sigma$ variation from the mean value. The $1 \sigma$ level was computed in this paper by selecting the regions by hand where no peak was visible. In the study presented here, we used a fully automated method, better suited to the large number of clusters to analyse. We ran the SExtractor software on plots of the number of galaxies versus the one-dimensional sector angle. The deblending threshold was set to 1, in order to favour the detection of major LSS extending over several angular sectors. The detection and analysis thresholds were set to 2 .

This procedure provided us with a number of significant detected galaxy concentrations (SDGC) around the T0006+Th09 sample of 491 clusters in the W1 field. We show an example in Fig. 19 of the SDGCs within a $2.5 \mathrm{Mpc}$ radius, detected for magnitude interval I.

\subsection{Reference sample}

We also defined a reference sample (i.e. galaxies that are not in clusters), in order to estimate the statistical behaviour of a random selection. We selected 491 random positions (both in RA, Dec and in redshift) in the same area as the real clusters and taken in interval I (see above). We then applied the same previous analysis and defined SDGCs around these random positions. We did not assign $S / N$ detections or $\mathrm{cD}$ galaxy magnitudes to these random positions, so of course only interval I can be compared to the random sample.

This allowed us to compute typical uncertainties on the SDGC numbers, computed as the second-order momenta of the SDGC distributions in the random sample computed across the field of view (in pixels of $0.2 \times 0.2 \mathrm{deg}^{2}$ ).

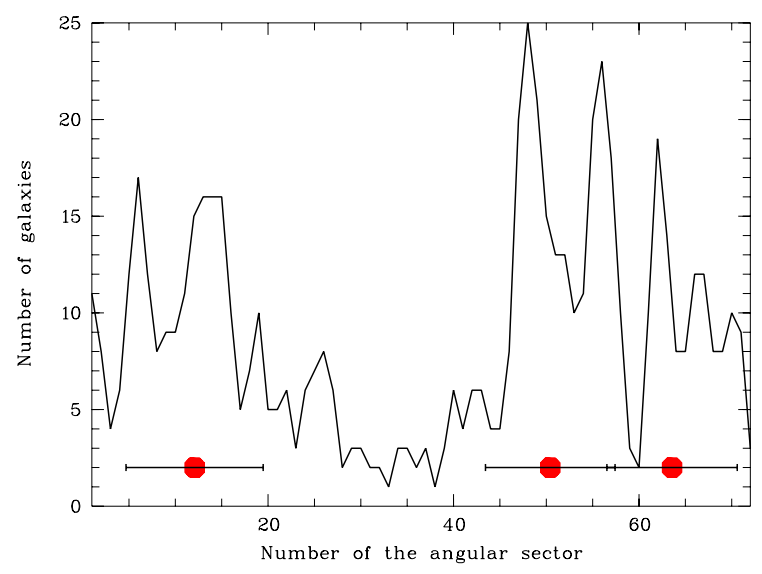

Fig. 19. Significant detected galaxy concentrations (SDGC) around one cluster when considering magnitude interval I within a $2.5 \mathrm{Mpc}$ radius from the cluster centre (see text). This figure shows the number of galaxies in $\mathrm{a} \pm 0.05$ photometric redshift bin around the considered cluster as a function of the sector number. Filled red circles: SDGC detected by SExtractor together with their $1 \sigma$ level extension. The definition of the angular sector is given in the text.

\subsection{Results}

The results of our analyses are shown in Figs. 20 and 21 (with error bars computed as standard deviations).

First, we can see that the mean number of SDGC decreases as a function of distance to the cluster centre for the interval I sample (upper to lower figures in Figs. 20 and 21). This result can be interpreted as a mean detection of numerous infalling structures at radius smaller than $1 \mathrm{Mpc}$ (the densest parts of the clusters). This is qualitatively consistent with the picture of a cluster drawn by the Millennium simulations (Springel et al. 2005). More distant areas would provide direct detections of cosmological filaments, in particular beyond the virial radius, but nothing significant is apparent in these areas. Our analyses at radii smaller than $1 \mathrm{Mpc}$ most of the time provide at least four directions with galaxy overdensities (see Figs. 20 and 21), suggesting infall rather than just cluster asymmetries. Moreover, we detect significantly more galaxy overdensities at radii smaller than $1 \mathrm{Mpc}$ in the real samples compared to the random samples. This also speaks in favour of a real trend.

Second, there is no strong variation in the mean SDGC value as a function of the cluster $S / N$ detection when we consider all the available magnitudes (sample I). However, high $S / N$ clusters (typically detections greater than $4 \sigma_{\mathrm{s}}$ ) exhibit lower mean SDGC values, in particular for the 1.0-3.5 Mpc corona, when considering the magnitude interval II (only bright galaxies). Clusters therefore seem to be statistically fed by a comparable number of filaments regardless of their richness. However, SDGCs populated with bright galaxies are less numerous beyond radii of $1 \mathrm{Mpc}$ in projection for rich clusters. This can be interpreted in a simple statistical way: it is not very likely that a rich cluster has a richer structure in its direct vicinity, while a poor cluster is more likely to have a rich companion.

Third, for radii smaller than $2.5 \mathrm{Mpc}$, we hardly detect any SDGC with bright galaxies (interval II) compared to the numbers detected in magnitude interval I. In contrast, nearly all SDGC are detected both in magnitude intervals I and II beyond radii of 2.5 Mpc. This is in good agreement with a general depopulation of bright galaxy structures in the immediate cluster vicinity, as already suggested by Adami et al. (2011). 

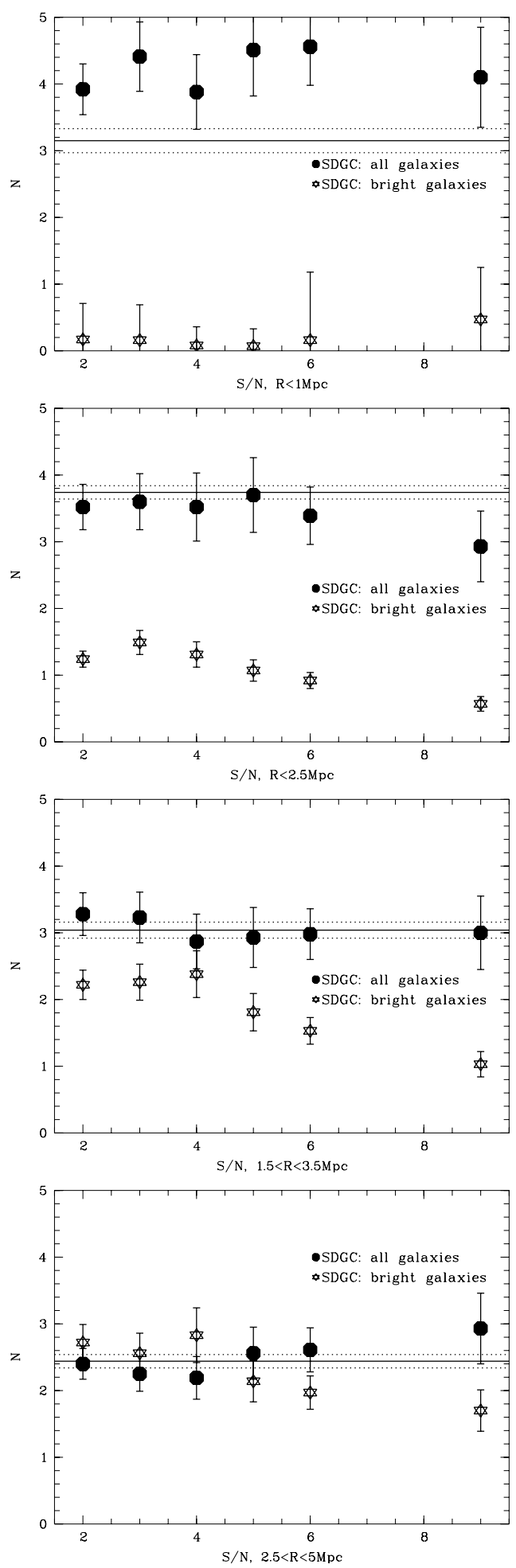

Fig. 20. Mean number of significant detected galaxy concentrations (SDGC) around the sample of 491 clusters in W1 from T0006+Th09 as a function of cluster detection level $(S / N)$ in the T0006 sample, in regions of different radii. From top to bottom the radii are: $R<1 \mathrm{Mpc}$, $R<2.5 \mathrm{Mpc}, 1.5<R<3.5 \mathrm{Mpc}$, and $2.5<R<5 \mathrm{Mpc}$. Filled circles: SDGC detected with galaxies in the largest possible magnitude interval (see text). Open stars: SDGC detected with galaxies brighter than the considered cluster cDs (see text). Continuous line: mean number of SDGC in the random sample with its error (dotted lines). The uncertainty (interval between the two dotted lines) is computed as the second-order momentum of the SDGC distribution across the field of view (in pixels of $0.2 \times 0.2 \mathrm{deg}^{2}$ ). By "all galaxies" and "bright galaxies" we mean galaxies belonging to intervals I and II, respectively.
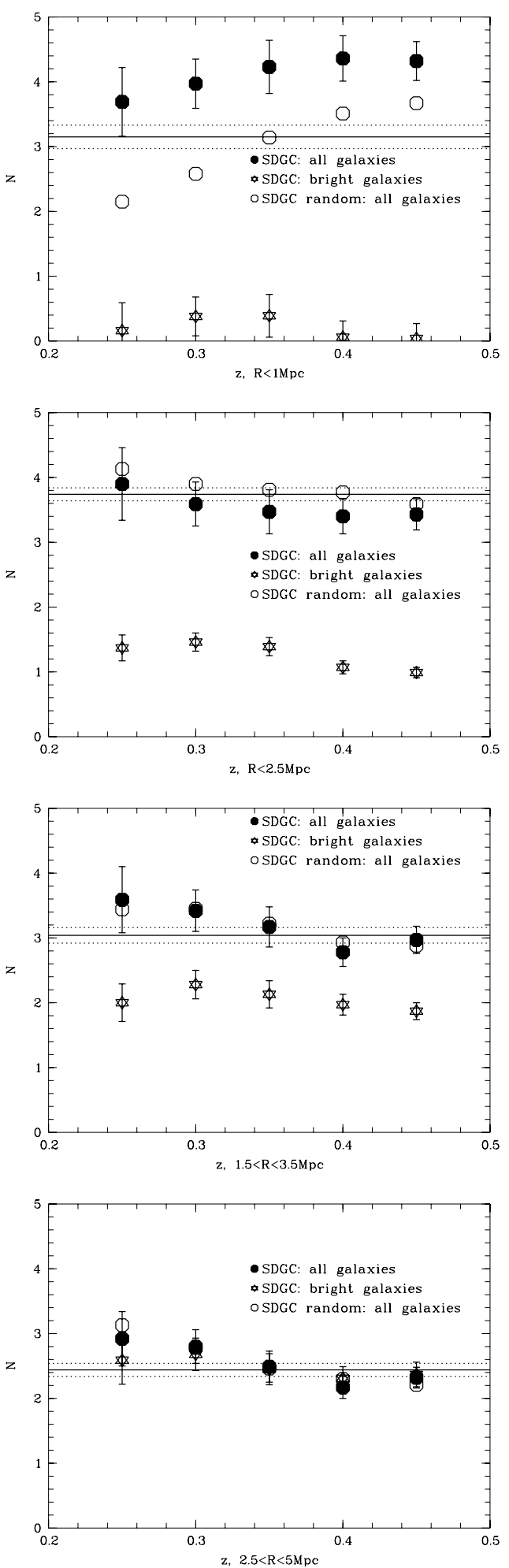

Fig. 21. Same as Fig. 20 but this time as a function of cluster redshift.

Fourth, we do not detect strong variations in the mean SDGC values as a function of redshift. Apparent variations seen in Fig. 21 are also visible for the random reference sample and are therefore probably coming from residual selection effects.

Fifth, we detect more SDGC at radii smaller than $1 \mathrm{Mpc}$ in the cluster sample than in the random reference sample. This behaviour is expected if our cluster sample really includes massive 
structures formed at the nodes of the cosmological filaments. Conversely, we detect slightly fewer SDGCs for radii smaller than $2.5 \mathrm{Mpc}$ in the cluster sample than in the random reference sample. This is also expected if, as previously suggested, clusters are depopulating their vicinity just beyond their densest areas. Finally, when considering areas at radii larger than the typical virial values, we have exactly the same mean SDGC values when considering the cluster or the random reference sample. This tendency is detected both as a function of the cluster detection $S / N$ and redshift. One more time, this is expected as we are dealing with areas where the clusters no longer have any influence.

\section{Discussion and conclusions}

We have searched for clusters of galaxies in the full CFHTLS Wide survey, based on the T0006 data release. Our method is based on the galaxy photometric redshifts computed with Le Phare, limited to magnitudes $z^{\prime} \leq 22.5$. We constructed galaxy density maps in photometric redshift bins of 0.1 based on an adaptive kernel technique, detected structures with SExtractor at various detection levels, and built cluster catalogues by applying a minimal spanning tree algorithm. We detected several thousand candidate clusters with estimated mean masses between $1.3 \times 10^{14}$ and $12.6 \times 10^{14} M_{\odot}$, thus increasing the number of known optical high-redshift cluster candidates by a large factor, an important step towards obtaining reliable cluster counts to measure cosmological parameters.

The cross-identification of the clusters in our catalogues with various other cluster searches in these regions based on other methods gives consistent results, in particular for clusters that we detect at $3 \sigma$ or more. By stacking a subsample of 45 clusters, we show that this stacked cluster indeed has "normal" cluster characteristics (colour-magnitude relation, galaxy luminosity function). The cluster-cluster correlation function is comparable to the one obtained in A10 (slope and amplitude).

We also analysed galaxy distributions in cosmological filaments, based on a sample of 491 clusters detected both by our method and by Thanjavur et al. (2009) in the W1 field. For these clusters, we counted the numbers of galaxies in 72 sector angles around the cluster and searched for significant peaks in these distributions. This procedure provided us with a number of SDGC. We find that the mean number of SDGC decreases as a function of distance to the cluster centre, as expected if we are detecting infalling structures at projected radii smaller than $1 \mathrm{Mpc}$ (the densest parts of the clusters). There is no strong variation in the mean SDGC value as a function of the SDGC $S / N$ detection, though high $S / N$ clusters tend to exhibit lower mean SDGC values. Clusters therefore seem to be statistically fed by a comparable number of filaments, regardless of their richness. However, SDGCs populated by bright galaxies are less numerous beyond radii of $1 \mathrm{Mpc}$ for rich clusters. We find a general depopulation of bright galaxy structures in the immediate cluster vicinity, as already found by Adami et al. (2011). We do not detect strong variations of the mean SDGC values as a function of redshift. We detected more SDGC at radii smaller than $1 \mathrm{Mpc}$ in the cluster sample than in the random reference sample and slightly fewer SDGCs for radii smaller than $2.5 \mathrm{Mpc}$, as expected if our cluster sample really includes massive structures formed at the nodes of the cosmological filaments, while clusters are depopulating their vicinity just beyond their densest areas.

The 3D spatial distributions of the candidate clusters detected in all the Wide fields show no obvious concentration of clusters or large-scale structures. In view of all the properties presented in this paper, our detected clusters behave as expected if they are located at the intersection of filaments by which they are fed.

A more detailed analysis of the properties of this sample of clusters and of the associated cosmological implications is beyond the scope of the present paper and will be done in a future work.

Note added in proof. During the refereeing process of the present paper, a search for clusters was made in several fields, including some in the CFHTLS Wide fields, and published by Wen \& Han (2011). The percentages of clusters that we redetect are comparable to those of the Thanjavur catalogues.

Acknowledgements. We acknowledge the referee's very thorough reading and constructive comments, which helped to improve the paper. I.M. acknowledges financial support from the Spanish grant AYA2010-15169 and Junta de Andalucía TIC114 and the Excellence Project P08-TIC-03531. We are grateful to K. Thanjavur for sending us his catalogues prior to publication. The Millennium Simulation databases used in this paper and the web application providing online access to them were constructed as part of the activities of the German Astrophysical Virtual Observatory.

\section{Appendix A: Galaxy selection}

The numbers of galaxies in the various catalogues used in this paper are given in Table A.1. We can see that the selection criterium based on the $z^{\prime}$ magnitude chosen here $\left(z^{\prime} \leq 22.5\right)$ gives numbers of galaxies comparable to those obtained with the criterium based on $i^{\prime}$ magnitudes by A10 $\left(0<i^{\prime} \leq 23\right)$, confirming that it is meaningful to compare the present results directly with those of A10. However, the $z^{\prime} \leq 22.5$ selection tends to make us lose low-redshift galaxies and gain few high-redshift galaxies, as discussed in Sect. 2.1.

Table A.1. Number of galaxies in the four Wide fields.

\begin{tabular}{lcccc}
\hline \hline & Wide 1 & Wide 2 & Wide 3 & Wide 4 \\
\hline Total number $(N)$ & 5346671 & 2100453 & 3858738 & 2040682 \\
$N\left(0<z^{\prime} \leq 22.5\right)$ & 2344677 & 1031367 & 1673192 & 1060507 \\
$N\left(0<i^{\prime} \leq 23\right.$ or $\left.0<y \leq 23\right)$ & 2658897 & 1137413 & 1900186 & 1162730 \\
\hline
\end{tabular}

Notes. Second line: galaxies with magnitudes $0<z^{\prime} \leq 22.5$, third line: galaxies with magnitudes $0<i^{\prime} \leq 23$ or $0<y \leq 23$.

\section{References}

Adami, C., \& Mazure, A. 1999, A\&AS, 134, 393

Adami, C., Le Brun, V., Biviano, A., et al. 2009, A\&A, 507, 1225

Adami, C., Durret, F., Benoist, C., et al. 2010, A\&A, 509, A81 (A10)

Adami, C., Mazure, A., Pierre, M., et al. 2011, A\&A, 526, A18

Ascaso, B., Wittman, D., Benítez, N., et al. 2010 [arXiv: 1011.3513]

Bahcall, J. N., \& Soneira, R. M. 1983, ApJ, 270, 20

Bergé, J., Pacaud, F., Réfrégier, A., et al. 2008, MNRAS, 385, 695

Bertin, E., \& Arnouts, S. 1996, A\&AS, 117, 393

Bielby, R. M., Finoguenov, A., Tanaka, M., et al. 2010, A\&A, 523, A66

Boué, G., Adami, C., Durret, F., Mamon, G., \& Cayatte, V. 2008, A\&A, 479, 335

Cabanac, R. A., Alard, C., Dantel-Fort, M., et al. 2007, A\&A, 461, 813

Cappi, A., \& Maurogordato, S. 1995, ApJ, 438, 507

Chilingarian, I. V., Melchior, A.-L., \& Zolotukhin, I. Yu. 2010, MNRAS, 405, 1409

Coupon, J., Ilbert, O., Kilbinger, M., et al. 2009, A\&A, 500, 981

Durret, F., Laganá, T. F., \& Haider, M. 2011, A\&A, 529, A38

Erben, T., Hildebrandt, H., Lerchster, M., et al. 2009, A\&A, 493, 1197 
Evrard, A. E., MacFarland, T. J., Couchman, H. M. P., et al. 2002, ApJ, 573, 7 Gavazzi, R., \& Soucail, G. 2007, A\&A, 462, 459

Geach, J. E., Murphy, D. N. A., \& Bower, R. G. 2011, MNRAS, 413, 3059

Gioia, I. M., Henry, J. P., Maccacaro, T., et al. 1990, ApJ, 356, L35

Goranova, Y., Hudelot, P., Magnard, F., et al. 2009, The CFHTLS T0006

Release, http://terapix.iap.fr/cplt/T0006-doc.pdf

Guennou, L., Adami, C., Ulmer, M. P., et al. 2010, A\&A, 523, A21

Grove, L. F., Benoist, C., \& Martel, F. 2009, A\&A, 494, 845

Hamilton, A. J. S. 1993, ApJ, 417, 19

Hildebrandt, H., Pielorz, J., Erben, T., et al. 2009, A\&A, 498, 725

Hildebrandt, H., Arnouts, S., Capak, P., et al. 2010, A\&A, 523, A31

Ilbert, O., Arnouts, S., McCracken, H. J., et al. 2006, A\&A, 457, 841

Juett, A. M., Davis, D. S., \& Mushotzky, R. 2010, ApJ, 709, L103

Klypin, A. A. \& Kopylov, A. I. 1983, SvA Lett., 9, 41

Labatie, A., Starck, J.-L., Lachièze-Rey, M., \& Arnalte-Mur, P. 2011, Statistical Methodology, in press [arXiv: 1009.1232]
Landy, S. D., \& Szalay, A. S. 1993, ApJ, 412, 64

Le Fèvre, O., Vettolani, G., Garilli, B., et al. 2005, A\&A, 439, 845

Limousin, M., Cabanac, R., Gavazzi, R., et al. 2009, A\&A, 502, 445

Mazure, A., Adami, C., Pierre, M., et al. 2007, A\&A, 467, 49 (M07)

Milkeraitis, M., Van Waerbeke, L., Heymans, C., et al. 2010, MNRAS, 406, 673

Mellier, Y., Bertin, E., Hudelot, P., et al. 2008, The CFHTLS T0005 Release, http://terapix.iap.fr/cplt/oldSite/Descart/ CFHTLS-T0005-Release.pdf

Olsen, L. F., Benoist, C., Cappi, A., et al. 2007, A\&A, 461, 81

Olsen, L. F., Benoist, C., Cappi, A., et al. 2008, A\&A, 478, 93

Romer, A. K., Viana, P. T. P., Liddle, A. R., \& Mann, R. G. 2001, ApJ, 547, 594

Springel, V., White, S. D. M., Jenkins, A., et al. 2005, Nature, 435, 629

Thanjavur, K., Willis, J., \& Crampton, D. 2009, ApJ, 706, 571

van Breukelen, C., \& Clewley, L. 2009, MNRAS, 395, 1845

Wright, E. L. 2006, PASP, 118, 1711

Wen, Z. L., \& Han, J. L. 2011, ApJ, 734, 68 